\title{
Advanced Adaptation Techniques for Improved Video Perception
}

\author{
G. Nur, H. Kodikara Arachchi, Member, IEEE, S. Dogan, Member, IEEE, and A. Kondoz, \\ Member, IEEE,
}

\begin{abstract}
Three different advanced adaptation techniques for improving the video perception of users are proposed in this paper. The proposed techniques exploit different adaptation decision taking and adaptation approaches to adapt particular core parameters while considering diverse contextual information and constraints to achieve improved video perception of users. The first proposed technique employs a utility-based adaptation approach to perform adaptation operations on spatial resolution, frame rate, and quality scalability parameters according to the content related contextual information (i.e., motion activity and structural feature) while fulfilling network bandwidth and terminal display size constraints. Using this technique, video contents can be adapted with the scalability parameters best fitting users' and contextual constraints' needs to achieve improved video perception. The second technique relies on prioritizing key frame, non-key frame, and temporal layer parameter related Network Abstraction Layer Units (NALUs) to adapt video contents to satisfy network bandwidth constraint. The Rate-Distortion (R-D) performances of adapted video contents can be improved by utilizing this technique in adaptation operations both in terms of bit rate of adapted video contents and video perception of users. The third technique is based on adapting the bit rate of 3-Dimensional (3D) video contents according to the changes in ambient illumination of the viewing environment. The adaptation results evaluated by either subjective or objective quality assessment techniques prove that all of the proposed techniques are efficient to improve the video perception of users.
\end{abstract}

Index Terms-adaptation decision taking, ambient illumination, NALU, SVC, UF, video adaptation, video perception

\section{INTRODUCTION}

$\mathrm{T}^{\mathrm{n}}$ he tremendous advances in video communication technology bring out the heterogeneity of the existing systems, services, applications, devices, etc. In light of these advances, there is an increase in the desire of users for better perceptual video quality regardless of contextual information (e.g., ambient illumination, motion activity of the video content, etc) and constraints (e.g., network bandwidth, display size, etc). Likewise, due to these advances, today's interactive video communication environment becomes very demanding for providing improved video perception of users and RateDistortion (R-D) performances of video contents. Therefore, it is important that user and performance friendly processes and

Manuscript received Jan.12, 2011; revised May 22, 2011.

Gokce Nur, Hemantha Kodikara Arachchi, Safak Dogan, and Ahmet M. Kondoz are with the I-Lab Multimedia Communications Research, Centre for Vision, Speech and Signal Processing, University of Surrey, Guildford, UK, (email: G.Nur, H.Kodikaraarachchi, S.Dogan, A.Kondoz@surrey.ac.uk). techniques are developed in response to this demand. Video adaptation is the most important process that provides an essential link between video contents, contextual information and constraints to yield improved video perception of users [1]. Therefore, advanced video adaptation techniques, which can react to the needs posed by the contextual information and constraints while adapting video contents, should be developed to achieve improved video perception of users.

Determining the shortcomings of the existing video adaptation techniques, which exploit different adaptation decision taking and adaptation approaches to improve video perception, is a tough nut to crack while devising advanced video adaptation techniques. Adaptation decision taking is a process of deciding the most important adaptation parameters to utilize in adaptation operations [2]. Thus, it can be considered as the 'brain' of the adaptation process. Likewise, core adaptation parameters (e.g., spatial resolution, key frame, etc), which can be considered as the fundamental elements of adaptation operations, should be ascertained to accomplish an important step towards developing advanced video adaptation techniques. In addition, to allow advanced video adaptation techniques to perform meaningful adaptation operations, it is imperative that core contextual information and constraints that can significantly affect adaptation decision taking processes are determined. Using the determined core adaptation parameters with different adaptation decision taking and adaptation approaches (e.g., prioritization-based, utilitybased, etc) advanced adaptation techniques can be developed to cope with the requirements of the varying contextual information and constraints. In this paper, three advanced video adaptation techniques, which adapt specific core adaptation parameters of scalable video contents in response to certain core contextual information and constraints by utilizing particular adaptation decision taking and adaptation approaches, are proposed to attain improved video perception of users.

The first advanced adaptation decision taking technique employs a utility-based adaptation decision taking approach to perform adaptation operations. Utility-based adaptation decision taking approach is based on unifying interrelation of video entities and three important spaces to address an optimisation problem, namely: adaptation, resource, and utility spaces. The interrelationship of adaptation operations, resources and quality values can be expressed with a Utility Function (UF) in this approach. This function is a model for measuring the utility of the video after undergoing adaptation operations. It has to be noted that there is an important difference between the terms utility and quality. Utility refers 
to the satisfaction of the end-user whereas quality refers to the quality of the content [1]. There are implementations of UFs in adaptation decision taking operations in literature. In [2], a simple UF, which only considers bit rate of video contents and is irrespective of video content characteristics, is used in adaptation decision taking operations. In [4], adaptation, resource, and utility aspects of adaptation strategies are modeled with a UF. The UF presented in this study considers the frame rate and spatial resolution, but not the quality in the adaptation operations. In [5], only a conceptual framework modeling the relationships among adaptation (e.g., video skimming, frame dropping, etc), resource (e.g., network bandwidth, display resolution, etc), and utility (e.g., objective or subjective quality of a video content) is developed. The objective of this framework is to find the optimal adaptation operations satisfying the resource constraints for achieving maximum utility for a given video content. In [6], a UF, which relies on the interrelationship of the spatial resolution, frame rate, and Quantization Parameter (QP), is designed. The importance of the spatial resolution, frame rate, and QP on the UF calculation with respect to each other is computed using weights. A recommendation system is used to maintain feedback from each user to determine the weights of the UF for each user. In [7], a UF that relies on jointly modeling the frame rate and spatial resolution is proposed. In addition, the frame rate and spatial resolution are considered as the functions of hardware capabilities (e.g., Whetstone metric, which is used to indicate the performance of the Central Processing Unit (CPU) of a computing device) and video content characteristics (e.g., low spatial details in the video sequence) in this study. Although the frame rate and spatial resolution are taken into consideration, the quality aspect of the video has not been considered in the UF. In [8], a UF, which models the bit rate, spatial resolution, quality of the video contents, and the terminal capabilities (i.e., the screen size and the clock speed of the CPU of the terminal) is proposed. The UF proposed in [8] is extended in [9] with the inclusion of the transmission channel capacity to the UF. However, both of the UF models in [8] and [9] do not consider the video content characteristics for deriving adaptation decisions. In [10], a utility-based adaptation decision taking technique selecting adaptation parameters considering usage environment constraints for dynamic adaptation of video sequences are proposed. Frame rate, spatial resolution, and quality scalability parameters are not considered in this technique either.

In light of these discussions, it can be summarized that the existing adaptation techniques relying on a utility-based adaptation approach do not consider either all of the scalability parameters or content related contextual information in adaptation decision taking operations. However, the UF presented in this paper models the relationships among the frame rate, spatial resolution, and visual quality parameters. In this way, the relations of trade-off among all of the scalability parameters are considered in the UF. Moreover, this UF addresses the problem of deriving adaptation decisions depending on video content characteristics. Motion activity and structural features are the primary content related contextual information considered in this technique. Similarly, network bandwidth and display size are also considered as contextual constraints in the technique.

A prioritization of progressively ordering spatial, temporal, and quality (Signal-to-Noise-Ratio (SNR)) layer related Network Abstraction Layer Units (NALUs) is utilized by the scalability extension of H.264/Advanced Video Coding (AVC) to meet network bandwidth constraints during adaptation processes [11]. Nevertheless, NALUs of every video content adapted using this progressive prioritization order may not present improved Rate-Distortion (R-D) performances due to the effects of different video content characteristics. In addition, the scalability extension of H.264/AVC can only facilitate a conventional adaptation approach, which is based on dropping quality (SNR) enhancement layer NALUs of key and non-key frames only in a symmetric way during an adaptation operation [12]. Nevertheless, as discussed in our previous study in [13], only symmetrically dropping NALUs from key and non-key frames cannot provide flexible video adaptations. Therefore, an adaptation approach, in which the quality (SNR) enhancement layer NALUs of key and non-key frames can be both symmetrically and asymmetrically dropped during an adaptation operation, is proposed in this paper. The second advanced adaptation decision taking technique proposed in the paper thus relies on the prioritization of key and non-key frame, and temporal layer NALUs to meet the network bandwidth contextual constraint considering the proposed adaptation approach. Due to using different temporal layers while developing the technique, the motion activities of video contents are exploited as core contextual information in this technique.

Last but not the least, using ambient illumination condition as core contextual information during video adaptation is an interesting topic. An advanced video adaptation decision taking technique is developed to adapt 3-Dimensional (3D) video sequences considering ambient illumination contextual information while preserving the perceptual quality experienced by users. Motion, structural feature, luminance contrast, and depth feature of 3D video sequences, and ambient illumination constitute the core contextual information in this technique.

This rest of the paper is organized as follows. Section II discusses the first advanced adaptation technique. The second advanced adaptation decision taking technique is described in Section III. The third advanced adaptation technique is introduced in Section IV. Section V presents the results of each of the techniques. The paper is concluded in Section VI.

\section{A UTILITY-BASED SCALABLE VIDEO ADAPTATION TECHNIQUE USING SPATIAL, TEMPORAL, AND QUALITY SCALABILITY PARAMETERS}

The Scalable Video Coding (SVC) standard, including the scalability extension of H.264/AVC [3], offers a comprehensive set of scalability parameters (e.g., spatial, temporal, and quality (SNR) scalabilities) to assist video adaptation. Selecting the best set of scalability parameters to be used in adaptation operations, while satisfying a set of contextual constraints for accomplishing enhanced video 
perception, is still a challenging issue despite the improvements in the scalable video adaptation.

When the contextual constraints such as the available network bandwidth are limited, at least one of the highest spatial resolution, frame rate, and quality parameters available in a scalable encoded sequence (or a combination of them) has to be sacrificed to meet this constraint. In order to achieve enhanced video perception, while sacrificing from these parameters, content related contextual information should be considered. For instance, sacrificing high spatial resolution rather than high frame rate would be a better adaptation solution for high motion video sequences to meet contextual constraints while still achieving enhanced video perception of users.

An advanced adaptation decision taking technique using a utility-based adaptation decision taking approach to carry out adaptation operations on spatial resolution, frame rate, and quality scalability parameters according to the content related contextual information while fulfilling network bandwidth and display size contextual constraints is developed, in this study. Adaptation Quality of Service (AQoS) and Universal Constraints Descriptor (UCD), which are MPEG-21 tools [14] are used for assisting video adaptation in this study. The framework of this advanced adaptation technique is presented in Fig.1. As realized from the figure, the framework is categorized into three main modules namely: AQoS, adaptation, and UCD. The heart of the framework is the adaptation module. This module includes the Adaptation Decision Taking Engine (ADTE) and the Adaptation Engine (AE) sub-modules to execute adaptation processes. The ADTE sub-module takes AQoS, which supplies content related metadata, and UCD, which represents constraints specifications, to make appropriate adaptation decisions in the form of adaptation parameters by solving the optimization problem, which will be discussed later. Thus, the ADTE submodule decides on the scalability parameters that can be sacrificed to fulfill the constraints, which are provided by $\mathrm{UCD}$, using the content related metadata provided by AQoS.

The stream that can be extracted from an encoded sequence is called as a scalable sub-stream in this paper. As seen from Fig. 1, the content related metadata described in AQoS includes scalable sub-streams' parameters (i.e., spatial resolution, frame rate, and quality), the Video Quality Metric (VQM) results of the scalable sub-streams, and a UF [2], which is a model for measuring utility of video contents, in this study. A UF, which models the relationships among the core adaptation parameters (i.e., spatial resolution, frame rate, and quality) and the weights specifying the relative importance of these parameters, is proposed in this study to measure utility of video sequences. Utility is the measure of satisfaction or experience of users when a video sequence that has a specific spatial resolution, frame rate, and quality is viewed [1]. The general definition of the proposed UF is:

$$
U F=W_{F} F+W_{S} S+W_{Q} Q
$$

where, $W_{F}+W_{S}+W_{Q}=1$, and $F$ is the frame rate, $S$ is the spatial resolution, $Q$ is the quality of a video sequence, which is the measure of quality degradation introduced to the video sequence in terms of distortion or artifacts. $W_{F}, W_{S}$, and $W_{Q}$ denote the weights that refer to the influence of the frame rate, spatial resolution, and quality, respectively, on the UF.

Determining the values of the weights for different video sequence types is essential to improve the perceived quality of adapted sequences. Thus, Motion Level (ML) and Structural Feature Level (SFL) driven classification algorithms are proposed to classify the video sequences into different types/classes in this study. In order to determine the weights for the video sequences belonging to each class, users' reactions towards the variation in the core adaptation parameters of video sequences are monitored in subjective experiments. The weights are ascertained by analyzing the results of the experiments using multiple regression analysis [15]. A weighting factors table is developed using the weights associated with each class. The ML and SFL classification blocks in the framework implement the ML and SFL classification algorithms, respectively. Additionally, the UF generator block of the framework includes the UF and weighting factors table. Following, if the AQoS descriptor of a video sequence is required for adaptation decision taking, the video sequence is first categorized into a class using the ML and SFL blocks of the framework. Then, the weighting factors table is used to determine the weights associated with its class, and these weights are assigned to the UF in the UF generator block. Simultaneously, the video sequence is encoded to produce sub-streams with different core adaptation parameter values. The quality parameter values of the sub-streams are measured with VQM [16]. In order to measure VQM grades of the sub-streams that have different spatial resolutions and frame rates, the original-uncompressed versions of the video sequences were spatially and/or temporally down-sampled.

The UF including the assigned weights, the core adaptation parameters of the sub-streams, and the VQM grades of the quality parameters of the sub-streams are input into the AQoS generator to produce the AQoS descriptor. It should be noted that the AQoS descriptor is prepared offline. Thus, the ML and SFL classification of a sequence, assigning the weights of the UF, encoding the sequence, and measuring the quality parameter values of the sub-streams using VQM are also performed offline. VQM has high computational complexity [16]. However, due to its offline calculation, computationally low complex on-the-fly adaptation operations can be devised using the proposed framework. The UCD module supplies the bandwidth of the network and the width and height of the user display constraints in this study. After the ADTE determines the most appropriate core adaptation parameters, they are passed to the AE to generate the adapted scalable stream.

A. Classification of Video Sequences

1) Motion Level Driven Classification

The pyramidal implementation of the Lucas and Kanade technique [17] was used to measure the optical flow in video sequences in this study. Since motion information is not evenly distributed to all parts of an image, the motion feature points should be determined to be used in the optical flow measurements. The Shi and Tomasi algorithm [18] which selects the corners of the objects as the prominent motion feature points is used in the optical flow measurements. 


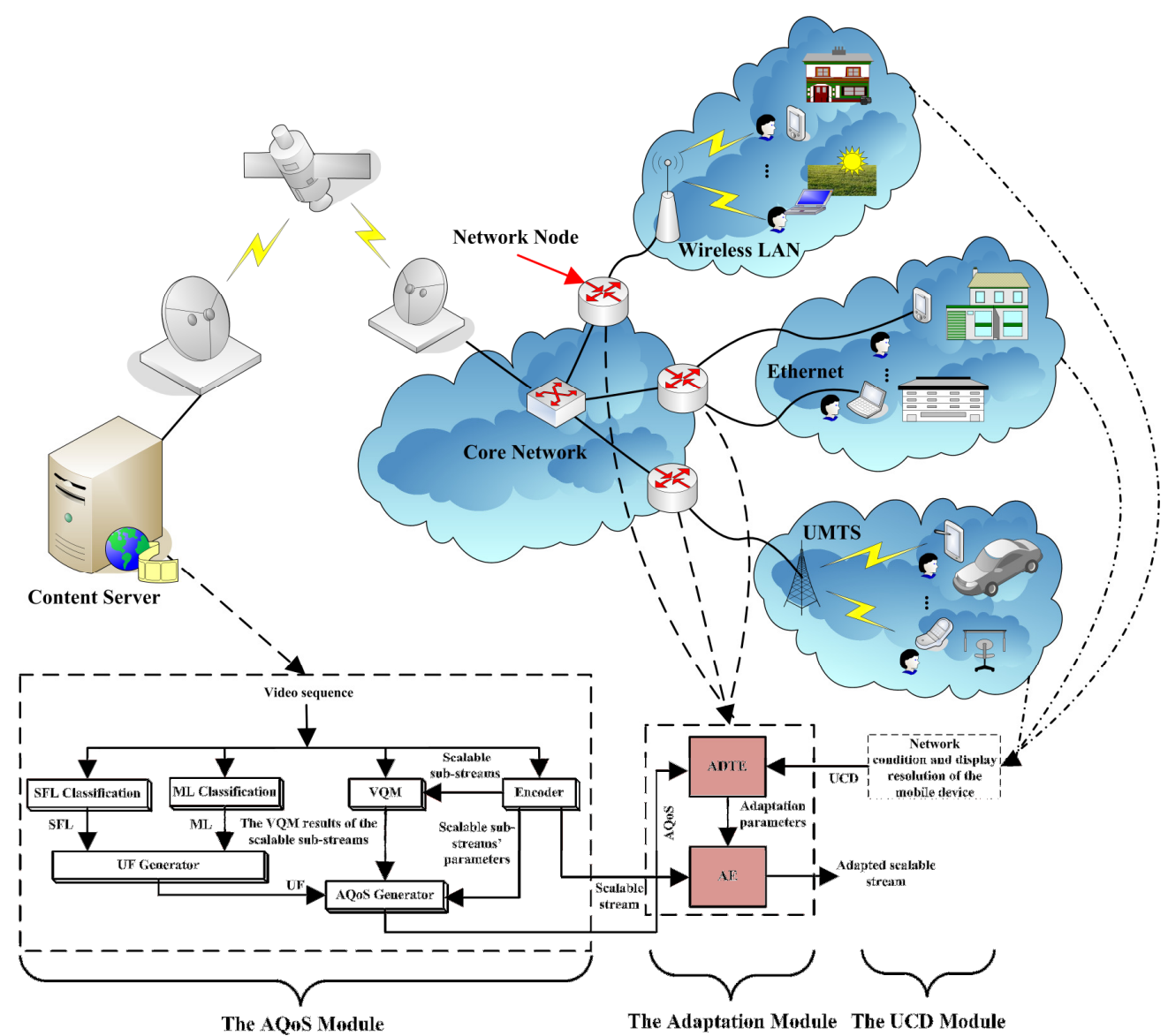

Fig. 1. The framework of the utility-based scalable video adaptation technique using spatial, temporal, and quality (SNR) scalabilities

After the feature points are determined, they are tracked from frame to frame in a video sequence by the pyramidal implementation of the Lucas and Kanade technique [17]. If $M V(x, y)$ is the motion vector of a feature point having $\mathrm{x}$ and $\mathrm{y}$ direction components, the motion intensity of a frame in a sequence is calculated as:

$$
\Pi(i)=\sum_{d=1}^{N o P}\left|M V_{d}\left(x_{i}, y_{i}\right)\right|
$$

where, $\Pi(i)$ is the motion intensity of the $i^{\text {th }}$ frame in the sequence. $d$ and $N o P$ are a feature point and the number of feature points in the frame, respectively. $M V_{d}\left(x_{i}, y_{i}\right)$ is the motion vector of the $i^{\text {th }}$ frame at feature point $\mathrm{d}$. A number of $400 \mathrm{NoPs}$, which is sufficient for achieving high feature point determination accuracy [19] are used in (2).

It should also be noted that the motion intensity of a frame is directly proportional to spatial resolution of the frame and inversely proportional to the temporal resolution of the video sequence. Therefore, the normalized average motion intensity over a given video sequence, which is used for ML classification, can be calculated as follows:

$$
M=\frac{\sum_{i=1}^{\mathrm{NOF}} \Pi(i)}{\mathrm{NoF}} \cdot \frac{F}{S}
$$

where, $M$ is the motion value in a sequence. $\mathrm{NoF}$ is the number of frames in the sequence. $F$ and $S$ are the frame rate and spatial resolutions of the sequence, respectively. In order to represent the $M$ results for different video sequences, (3) was tested with 18 video sequences, which have Common Intermediate Format (CIF: $352 \times 288$ pixels) resolution and 30 fps. Fig. 2 presents the $M$ results for these sequences.
The behavior of $M$ against various spatial and temporal resolutions of a selected sequence (i.e., Soccer) is demonstrated in Fig. 3 to prove that (3) provides similar results for video sequences that have different spatial resolutions (i.e., Standard Definition (SD: 704x576 pixels), CIF, and Quarter CIF (QCIF: 176x144 pixels)) and frame rates (i.e., 7.5, 15, and $30 \mathrm{fps}$ ).

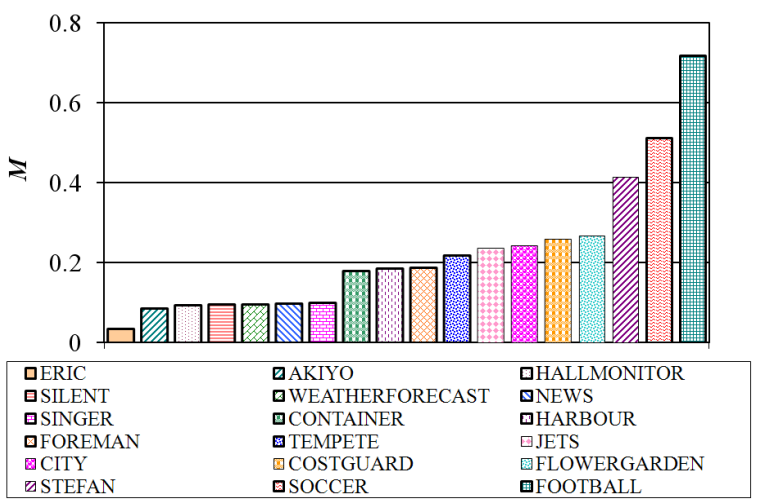

Fig. 2. The measured $M$ results for different video sequences

As also reported in [20], four different classes are determined to categorize the video sequences considering object and camera activities in this study. These four classes are: low motion, low-medium motion, medium-high motion, and high motion, as illustrated in Table I. In this table, the ML classes are represented with $\mathrm{A}, \mathrm{B}, \mathrm{C}$, and D. The $K$-median clustering method [21] is used to determine the borders for 
each class as also presented in Table I and the video sequences are classified into these four classes according to which border its $M$ values belongs to.

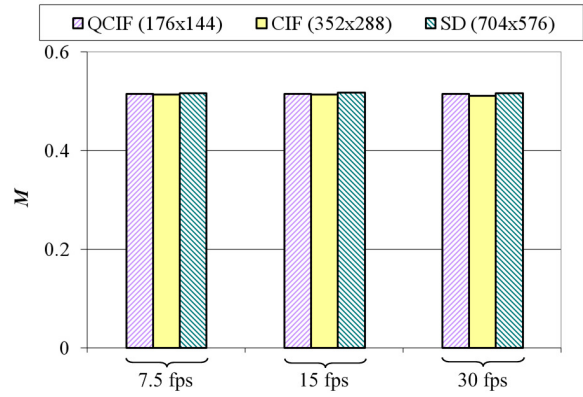

Fig. 3. The $M$ results for the Soccer sequence that has three different spatial resolutions (QCIF, CIF, and SD) and frame rates (7.5, 15, and $30 \mathrm{fps}$ ). TABLE I

MOTION LEVELS

\begin{tabular}{|c|c|c|c|}
\hline ML & Motion Intensity & Camera-Object(s) Motion & $M$ Values \\
\hline A & Low & Static-Static & $0<M \leq 0.1$ \\
\hline B & Low-Medium & Static-Moving & $0.1<M \leq 0.2$ \\
\hline C & Medium-High & Moving-Static & $0.2<M \leq 0.3$ \\
\hline D & High & Moving-Moving & $M>0.3$ \\
\hline
\end{tabular}

2) Structural Feature Level Driven Classification

The Canny edge detection algorithm [22] is used to determine the contour feature, which is associated with the structural feature, of the frames in this study. In order to measure contour density, the number of pixels that are set to 1 is counted in every frame of a video sequence. The total is then normalized using the NoF and $S$ to provide consistency across different video sequences, as follows:

$$
C=\frac{\sum_{i=1}^{\mathrm{NOF}} \delta(i)}{\mathrm{NoF} . S}
$$

where, $C$ is the structural feature value of a video sequence, which is used for SFL classification. $\delta$ is the number of edge pixels in the $i^{\text {th }}$ frame of the sequence. (4) was tested with different video sequences that have CIF resolution and $30 \mathrm{fps}$ as shown in Fig. 4. In order to prove that (4) provides similar $C$ results for video sequences that have different spatial resolutions and frame rates, the $C$ results of a selected sequence (i.e., Soccer) are illustrated in Fig. 5.

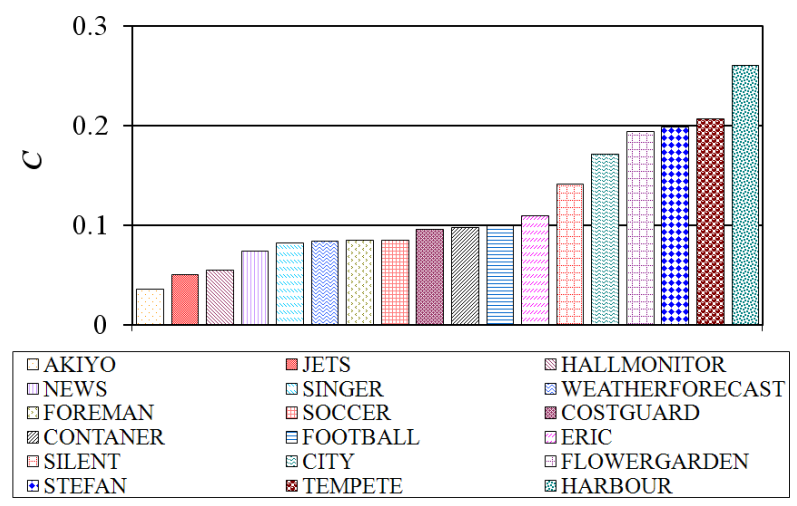

Fig. 4. The measured $C$ results for different video sequences

In this study, the SFLs were split into two generic categories: low and high complex. As shown in Table II, these classes are represented with $\mathrm{X}$ and $\mathrm{Y}$, respectively. Similar to the ML classes, $K$-median clustering method [21] is used to ascertain the borders for each of the classes, which are also shown in Table II. A video sequence can be classified into these two classes considering the borders of the $C$ values. Due to the cross matching of the MLs and SFLs presented in Table I and II, the video sequences are classified into eight classes as shown in Table III.

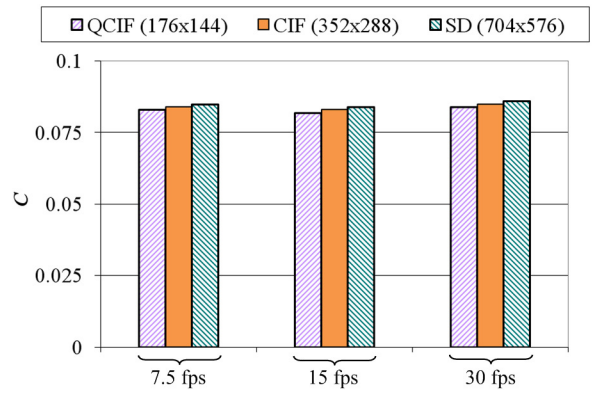

Fig. 5. The $C$ results for the Soccer sequence that has three different spatial resolutions (QCIF, CIF, and SD) and frame rates (7.5, 15, and $30 \mathrm{fps}$ ). TABLE II

STRUCTURAL FEATURE LEVELS

\begin{tabular}{|c|c|c|}
\hline SFL & Structural Feature & $C$ values \\
\hline $\mathrm{X}$ & Low Complex & $0<C \leq 0.1$ \\
\hline $\mathrm{Y}$ & High Complex & $C>0.1$ \\
\hline \multicolumn{3}{|c|}{ TABLE III }
\end{tabular}

CLASSES OF VIDEO SEQUENCES

\begin{tabular}{|c|c|c|c|c|c|c|c|c|}
\hline ML and SFL Class & 1 & 2 & 3 & 4 & 5 & 6 & 7 & 8 \\
\hline ML & A & B & C & D & A & B & C & D \\
\hline SFL & X & X & X & X & Y & Y & Y & Y \\
\hline
\end{tabular}

B. Subjective Experiments

Eight test sequences namely; Eric, Container, Jets, Soccer, Silent, Harbor, City, and Stefan were used in the subjective experiments, and each sequence was a representation of the video sequence classes ranging from 1 to 8 respectively (see Table III). Each of the test sequences were encoded with four different frame rates (i.e., 30, 15, 7.5, and $3.75 \mathrm{fps}$ ), three different spatial resolutions (i.e., SD, CIF, and QCIF) using JSVM 9.13.1 [23]. Seven different channel bandwidth values (i.e., 96, 128, 192, 256, 384, 512, and $768 \mathrm{kbps)} \mathrm{were} \mathrm{selected}$ as the target source rates. In order to match the bit rates of the encoded video sequences to the target source rates, different constant QP sets were utilized to encode the base and spatial enhancement layers. The sets producing sub-streams best matching with the target source rates were exploited to encode each sequence. Scalable sub-streams that have the target source rates were extracted from the encoded sequences for the experiments. The Double Stimulus Impairment Scale (DSIS) methodology [24] was used throughout the experiments. Thus, the test sequences were presented in pairs. The first sequence was always the original sequence, and the second one was the impaired version of the original sequence in this method. The subjects were asked to rate the impaired sequence with respect to the original. A 23"-Dell display was used during the experiments to display the sequences. The resolution of the display was $1680 \times 1050$ pixels. The video clips were displayed in their original sizes (i.e., SD, CIF, and $\mathrm{QCIF}$ ) and they are not up-sampled to SD resolution. The reason is that the aim of the subjective experiments is to monitor the spatial resolution, frame rare, and quality preferences of the users towards video sequences belonging to each ML and SFL class. A gray scale video was used to fill in the gap for the remaining parts of the display. The viewing distance for the observers was not fixed. However, it was kept 
in compliance with the Preferred Viewing Distance (PVD) of the ITU-R BT.500.11 [24]. 98 viewers (42 females and 56 males) participated in the experiments. Their ages ranged from 18 to 40 , with an average of 27 . None of the observers was previously involved in picture quality evaluations as part of their work, and they were not experienced assessors. They all reported normal and corrected eyesight. Owing to the large volume of the test sequences, the sequences were split into 12 groups, each consisted of 56 different video clips. 18 subjects assessed each group. Some of the subjects participated in more than one group. 2 observers were detected as outliers from each group using the outlier screening method discussed in [25]. Thus, in total 24 observers were detected as outliers, and the results of 74 viewers ( 33 females and 41 males) were used in the experiments. After the experiments, the Mean Opinion Scores (MOSs) and confidence intervals [24] were computed. After the outliers were eliminated, 16 subjects were used to calculate the MOSs in each group.

\section{Analysis of the Subjective Experiments Results}

In this section, the subjective test results are analyzed, so as to specify the weights of the core adaptation parameters in the UF (i.e., (1)). The frame rate preferences of the viewers towards Silent (i.e., ML and SFL Class 5), Container (i.e., ML and SFL Class 2), Jets (i.e., ML and SFL Class 3), and Stefan (i.e., ML and SFL Class 8) sequences encoded at 96-768 kbps are illustrated together with confidence intervals of the MOSs in Fig. 6. As can be realized from the figure, the preferences differ for the sequences due to their motion activity and structural features. The following findings are observed for the relationships among frame rate preferences of users, and motion and structural features of video sequences:

- The lower the frame rate, the higher the per frame quality is for the video sequences encoded at a given bit rate,

- When a video sequence has high motion activity, the viewers prefer high frame rate video representations since they present the real-world highly active scenes more naturally,

- When a video sequence presents a low structural feature, the details in the frames of a sequence require fewer bits compared to a high structural feature video sequence to have similar video perception at the same frame rate

The spatial resolution preferences of the viewers are illustrated in Fig. 7 for the Silent, Container, Jets, and Stefan sequences. The confidence interval values of the MOSs are also illustrated in the figure. As with the frame rate preferences, the spatial resolution preferences change according to the motion activity and structural feature characteristics of the sequences. The following observations are made for the relationship among the spatial resolution preferences of users and the motion activity and structural feature characteristics of video sequences:

- When the motion activity of a video sequence is high, it requires higher bit rates to achieve a similar video perception level with another sequence, which has a low motion activity, at the same spatial resolution representations,

- When a video sequence presents a high structural feature, the higher the spatial resolutions in which the structural details in a scene can be visualized the better the video perception of viewers is,

- The smaller the spatial resolution the higher the per pixel video quality is for video sequences encoded at a low given bit rate for medium-high and high motion video sequences

The results of the subjective experiments are analyzed with multiple regression analysis [15] to determine the weights of the UF (i.e., (1)) for each video sequence type. Regression equation models represent the dependent variables as functions of the independent variables. Here, the dependent variable is the UF, and the independent variables are the frame rate, spatial resolution, and quality. Accordingly, the general multiple regression equation is expressed as:

$$
U F^{\prime}=\widehat{W}_{F} \hat{F}+\widehat{W}_{S} \widehat{S}+\widehat{W}_{Q} \hat{Q}
$$

where $\hat{F}, \hat{S}$, and $\hat{Q}$ represent the predictor variables of frame rate, spatial resolution, and quality, respectively. $\widehat{W}_{F}, \widehat{W}_{S}$, and $\widehat{W}_{Q}$ are the regression coefficients of the unknown parameters, $W_{F}^{\prime}, W_{S}^{\prime}$, and $W_{Q}^{\prime}$. The objective here is to estimate the unknown parameters and then map the estimated values to the regression coefficients in (1). In order to avoid confusion between the parameters of the proposed UF equation in (1) and the multiple regression equation in (4), $U F$, $W_{F}, W_{S}$, and $W_{Q}$ in (1) are denoted as $U F^{\prime}, \hat{W}_{F}, \hat{W}_{s}$, and $\hat{W}_{Q}$ in (4), respectively. The results obtained from the subjective experiments were separated into 84 sets that have four components each: $\left(F_{1}, S_{1}, Q_{1}, M O S_{1}\right),\left(F_{2}, S_{2}, Q_{2}, M O S_{2}\right), \ldots$, $\left(F_{84}, S_{84}, Q_{84}, M O S_{84}\right)$. Here, $F, S, Q$, and $M O S$ represent the frame rate, spatial resolution, quality values, and the average MOS calculated following the experiments, respectively. The subscripts of the $F, S, Q$, and $M O S$ represent the sequence number in the sets. 84 sets were prepared since the combination of four frame rates (i.e., 3.75, 7.5, 15, and 30 fps), three spatial resolutions (i.e., QCIF, CIF, and SD), and seven average quality values associated with seven bit rates (i.e., 96, 128, 192, 256, 384, 512, and $768 \mathrm{kbps}$ ) used during the subjective tests for each video sequence type was $4 \times 3 \times 7$ $=84$. These sets were prepared for every video sequence type separately. In order to estimate the regression coefficients in (4), the following equation is formulated based on the multiple regression analysis [15] firstly:

$$
f\left(W_{F}^{\prime}+W_{S}^{\prime}+W_{Q}^{\prime}\right)=\sum_{j}\left[M O S_{j}-\left(W_{F}^{\prime} F_{j}+W_{S}^{\prime} S_{j}+W_{Q}^{\prime} Q_{j}\right)\right]^{2}
$$

where $j$ represents the number of sets each of which have four components (i.e., $F, S, Q$, and $M O S$ ). Thereafter, the partial derivative of $f$ is taken with respect to $W_{F}^{\prime}, W_{S}^{\prime}$, and $W_{Q}^{\prime}$. Then, all of the partial derivatives are equated to zero to yield the equations below:

$$
\begin{gathered}
W_{F}^{\prime} \sum_{j=1}^{84} F_{j}+W_{S}^{\prime} \sum_{j=1}^{84} S_{j}+W_{Q}^{\prime} \sum_{j=1}^{84} Q_{j}=\sum_{j=1}^{84} \operatorname{MOS}_{j} \\
W_{F}^{\prime} \sum_{j=1}^{84}\left(F_{j}\right)^{2}+W_{S}^{\prime} \sum_{j=1}^{84} S_{j} F_{j}+W_{Q}^{\prime} \sum_{j=1}^{84} Q_{j} F_{j}=\sum_{j=1}^{84} M O S_{j} F_{j} \\
W_{F}^{\prime} \sum_{j=1}^{84} F_{j} S_{j}+W_{S}^{\prime} \sum_{j=1}^{84}\left(S_{j}\right)^{2}+W_{Q}^{\prime} \sum_{j=1}^{84} Q_{j} S_{j}=\sum_{j=1}^{84} M O S_{j} S_{j} \\
W_{F}^{\prime} \sum_{j=1}^{84} F_{j} Q_{j}+W_{S}^{\prime} \sum_{j=1}^{84} S_{j} Q_{j}+W_{Q}^{\prime}+\sum_{j=1}^{84}\left(Q_{j}\right)^{2}=\sum_{j=1}^{84} M O S_{j} Q_{j}
\end{gathered}
$$

These equations are solved by computing all of the 
constants related to $F, S$, and $Q$, and then using the Gaussian function [15] as the elimination technique to determine the unknown parameters (i.e., $W_{F}^{\prime}, W_{S}^{\prime}$, and $W_{Q}^{\prime}$ ). Once these parameters were found, they were mapped to the regression coefficients (i.e., $\widehat{W}_{F}, \widehat{W}_{S}$, and $\widehat{W}_{Q}$ ). The results of the multiple regression analysis were then used to develop a weighting factors table including the weights of the UF for each ML and SFL class, as presented in Table IV.

TABLE IV

THE WEIGHTING FACTORS TABLE

\begin{tabular}{|c|c|c|c|}
\hline \multirow{2}{*}{ ML and SFL Class } & \multicolumn{3}{|c|}{ UF Weights } \\
\cline { 2 - 4 } & $W_{F}$ & $W_{S}$ & $W_{Q}$ \\
\hline 1 & 0.23 & 0.40 & 0.37 \\
\hline 2 & 0.31 & 0.36 & 0.33 \\
\hline 3 & 0.43 & 0.31 & 0.26 \\
\hline 4 & 0.46 & 0.30 & 0.24 \\
\hline 5 & 0.22 & 0.43 & 0.35 \\
\hline 6 & 0.30 & 0.39 & 0.31 \\
\hline 7 & 0.42 & 0.35 & 0.23 \\
\hline 8 & 0.45 & 0.34 & 0.21 \\
\hline
\end{tabular}

It should be noted that MOS is used to identify the weights of the UF for each ML and SFL class rather than the VQM in this paper. The reason is that VQM cannot reliably compute

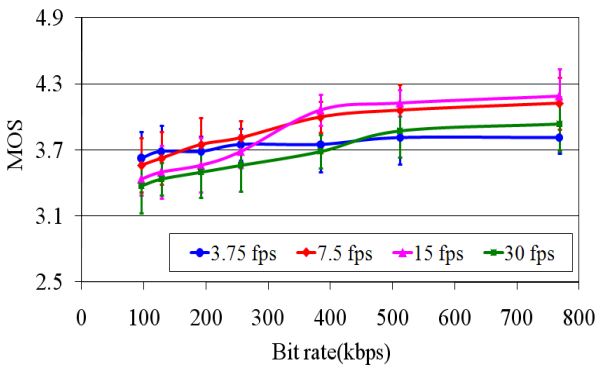

(a)

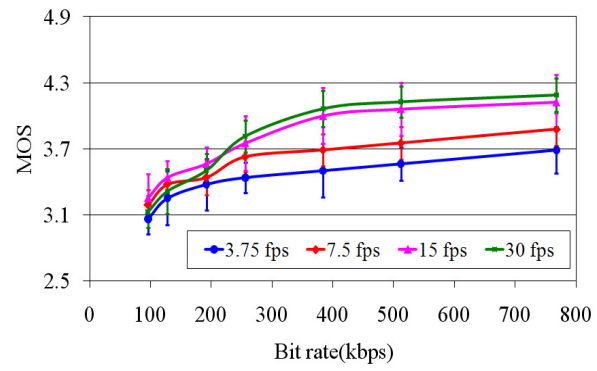

(c) the effects of different spatial resolution, frame rate, and quality parameters on video quality perception for the sequences belonging to each ML and SFL class. Therefore, human observers who are the "true assessors" of video quality perception are used to compute the MOS results. In this way, the weights of the UF are computed in the most reliable way using the subjective experiments.

D. Adaptation Decision Taking Process

The ADTE presented in Fig. 1 determines the most appropriate adaptation parameters by solving the following optimization problem:

Maximize: $\left\{U F=W_{F} F+W_{S} S+W_{Q} Q\right\} \Rightarrow$ Optimization Constraint Subject to: $\{$ bit rate $\leq$ target bit rate; width $\leq$ display width; height $\leq$ display height $\}=>$ Limitation Constraint

where, the optimization constraint represents the maximization of the UF (see (1)), bit rate is the bit rate of the scalable substream, target bit rate is the network bandwidth, width and height are the horizontal and vertical dimensions of the scalable sub-stream resolutions, respectively. Display width and height are respectively the horizontal and vertical dimensions of the terminal display resolution.

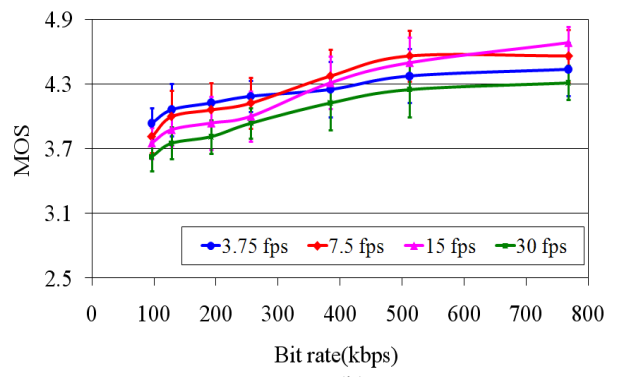

(b)

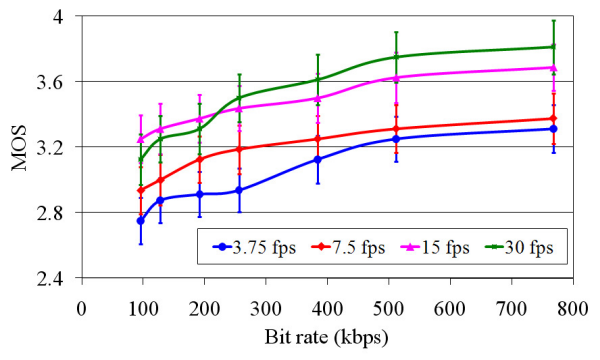

(d)

Fig. 6. MOS versus bit rates based on various frame rates for the (a) Silent (b) Container (c) Jets (d) Stefan sequences together with confidence intervals-

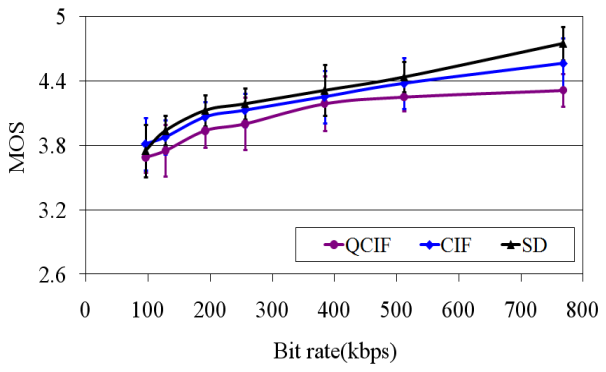

(a)

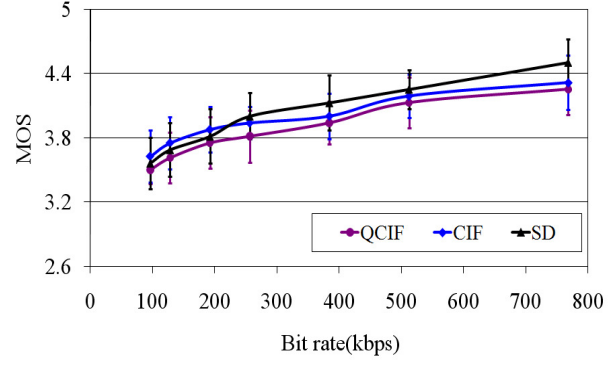

(b) 


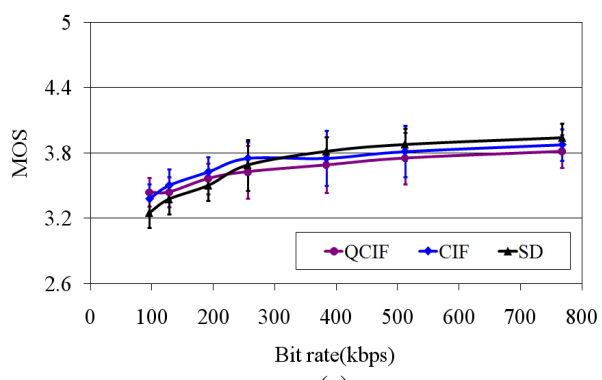

(c)

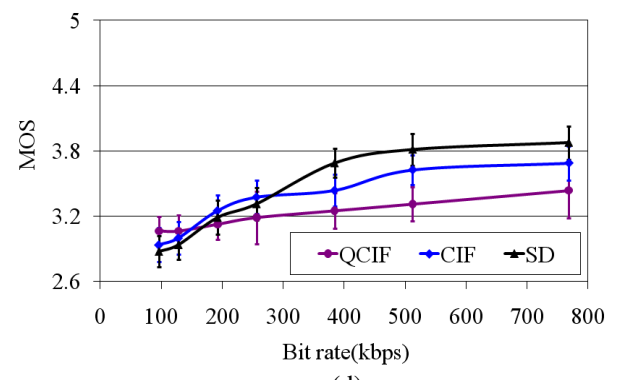

(d)

Fig. 7. MOS versus bit rates based on various spatial resolutions for the (a) Silent (b) Container (c) Jets (d) Stefan sequences together with confidence interval

III. A VIDEO ADAPTATION TECHNIQUE USING SYMMETRIC AND ASYMMETRIC KEY/NON-KEY FRAME QUALITY SCALABILITY ORDERING BASED PRIORITIZATION

The scalability extension of H.264/AVC uses a NALU prioritization, which relies on progressively ordering spatial, temporal, and quality (SNR) related NALUs until the target bit rate of the current network is achieved during an adaptation operation [11]. However, NALUs of video contents of every video sequence type adapted using this progressive prioritization order may not provide improved R-D performances. For example, NALUs of the temporal enhancement layers assigned higher priorities than those of the spatial enhancement layers would result in improved video perception for high motion video sequence types but low video perception for low motion video sequence types after adaptation operations. Therefore, the prioritization order should be modified according to the type of video sequences.

Moreover, the scalability extension of H.264/AVC supports a conventional adaptation approach, which is based on dropping quality (SNR) enhancement layer key and non-key frame NALUs in a symmetric way during adaptation operations [12]. Here, frames in the coarsest temporal layers are referred to as key frames, and all of the other frames between the key frames are referred to as non-key frames. The "key frame" concept is introduced by the Medium Grain Scalability (MGS) to adjust the balance between the drift and enhancement layer coding efficiency. Drift is observed if the motion-compensated prediction loops at the encoder and decoder are not synchronized. MGS enables the base layer motion-compensation only within the key frames so that drift is not observed for them. Moreover, in contrast, all of the nonkey frames use the highest available quality layer for motion compensation, which results in a higher R-D efficiency for these frames [3]. The conventional adaptation approach cannot provide flexibility to video adaptation operations since NALUs are dropped only in symmetrical way. Therefore, we propose to develop an adaptation technique in which the quality enhancement layer key and non-key frame NALUs can be both symmetrically and asymmetrically dropped. The achievable bit rate options for video adaptation operations increase using this proposed adaptation approach. In this way, the adverse effects of the network congestion can be decreased while ensuring better R-D performances compared to the conventional adaptation approach [13].

Adaptation experiments are conducted by applying the proposed adaptation approach for video sequences having different frame rates to assess the R-D performance of adapted video sequences. Accordingly, the core adaptation parameters that are used to develop the advanced adaptation technique discussed in this study are determined as: key frames, non-key frames, and frame rates. The video sequences used in the experiments belong to different MLs (see sub-section II/A/1). In this way, the motion activity of the video sequences is utilized as the core content related contextual information in this study. VQM is used as the quality metric during the R-D assessments correlation with the HVS [16]. Considering the knowledge gained through the R-D assessments, prioritization schemes each of which corresponds to a ML type to assist an encoder to assign a priority level for each NALU of encoded video sequences are developed. In this way, the advanced video adaptation technique of this study relies on using the proposed prioritization schemes for adaptation operations to achieve improved video perception of users and R-D performances of adapted sequences.

\section{A. Adaptation Experiments Using the Proposed Adaptation Approach}

Twelve sequences (i.e., Akiyo, News, Weather Forecast, Foreman, Harbor, Big Ships, Coastguard, Flower Garden, Tempete, Football, Stefan, and Tennis) having different motion activities were used to assess the proposed adaptation approach. The video sequences were at CIF resolution. All of the sequences were encoded in a scalable format with a base layer (level 0), and two MGS stacks each having two quality enhancement layers (i.e., four quality enhancement layers in total (levels 1, 2, 3, and 4)), with a Group of Picture (GOP) size of 4 and at $30 \mathrm{fps}$, using the JSVM 9.13.1 codec [23]. The frequency of the key frames was four since the GOP size was set to 4 in the sequences. A QP of 40, 35, and 30 were used to encode the base and two MGS stacks of the video sequences, respectively. Quality (SNR) scalability was achieved using the MGS technique [12].

In order to perform adaptation operations with all of the possible key and non-key frame variations of the encoded video sequences, the number of the base layer (i.e., 0) and quality enhancement layers of key frames (i.e., 1, 2, 3, 4) are cross-matched with those of the non-key frames. Each of the resultant cross-matches is called as a Variation (V) in this paper. All of the resultant Vs after the cross-matching are illustrated in Table V. Each of the encoded video sequences is then adapted considering each of these $\mathrm{Vs}$ and three different frame rates (i.e., 7.5, 15, and $30 \mathrm{fps}$ ). The qualities of these adapted sequences are then evaluated using VQM [16]. In order to measure the VQM of the adapted video sequences that have three different frame rates, the original video sequences 
are temporally down-sampled to these frame rates. VQM is a computationally high complex objective quality measurement metric [16]. Nevertheless, the qualities of the adapted sequences are offline measured using VQM. Thus, its high computational complexity does not have any effect on the complexity of the proposed technique.

Fig. 8 presents the VQM versus bit rate results at 7.5, $15 \mathrm{fps}$ and $30 \mathrm{fps}$ for Weather Forecast (i.e., ML A), Big Ships (i.e., ML B), Tempete (i.e., ML C), and Tennis (i.e., ML D) sequences, respectively. As can be seen from each of the graph in the figure, five Vs form each curve. Each point on a curve represents a video sequence adapted using a V (i.e., V1-V25) of Table $\mathrm{V}$ and a frame rate (i.e., 7.5, 15, or $30 \mathrm{fps}$ ). As can be observed from the figure, several points on the curves have similar VQM grades at different bit rates (e.g., "a" and "b" in Fig. 8 (a)). According to this observation, a significant amount of bit rate can be saved by adapting a sequence using the $\mathrm{V}$ and frame rate of adequate points (e.g., "a" instead of "b"). Additionally, several points on the curves have similar bit rates versus different VQM grades (e.g., "c" and "d" in Fig. 8 (a)).

Owing to this observation, it can be realized that achieving a better quality value at the same bit rates requires selecting the $\mathrm{V}$ and frame rate of efficient points (e.g., "c" instead of "d"). These kinds of observations can be made for the points on the curves of the other graphs as shown in Fig. 8. As a result, designing prioritization schemes for MLs based on this adaptation approach requires an R-D performance analysis using the points on the graphs of adapted sequences. TABLE V

VARIATIONS IN KEY AND NON-KEY FRAMES

\begin{tabular}{|c|c|c|c|c|c|c|c|c|c|c|c|c|c|c|c|c|c|c|c|c|c|c|c|c|c|}
\hline Variations & 〉 & $\stackrel{1}{>}$ & $\stackrel{n}{>}$ & $>^{+}$ & $\stackrel{n}{>}$ & $\stackrel{0}{>}$ & 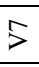 & $\stackrel{\infty}{>}$ & 9 & $\stackrel{\circ}{\circ}$ & $\bar{z}$ & $\frac{1}{>}$ & $\frac{m}{2}$ & $\stackrel{ \pm}{>}$ & $\frac{n}{2}$ & $\frac{0}{>}$ & $\frac{1}{>}$ & $\frac{\infty}{>}$ & $\frac{9}{2}$ & 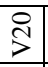 & \begin{tabular}{|l|l}
$\bar{\lambda}$ \\
\end{tabular} & $\begin{array}{l}> \\
\\
\end{array}$ & $\stackrel{3}{>}$ & \begin{tabular}{|l|l} 
\\
\end{tabular} & $\stackrel{2}{2}$ \\
\hline $\begin{array}{l}\text { The Number of Base and Enhancement Layers } \\
\text { of Key Frames }\end{array}$ & 4 & 4 & 4 & 4 & 4 & 3 & 3 & 3 & 3 & 3 & 2 & 2 & 2 & 2 & 2 & 1 & 1 & 1 & 1 & 1 & 0 & 0 & 0 & 0 & 0 \\
\hline $\begin{array}{l}\text { The Number of Base and Enhancement Layers } \\
\text { of Non-Key Frames }\end{array}$ & 4 & 3 & 2 & 1 & 0 & 4 & 3 & 2 & 1 & 0 & 4 & 3 & 2 & 1 & 0 & 4 & 3 & 2 & 1 & 0 & 4 & 3 & 2 & 1 & 0 \\
\hline
\end{tabular}

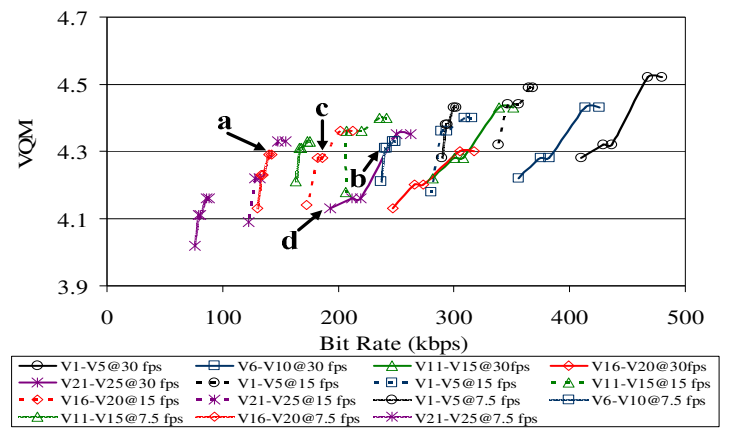

(a)

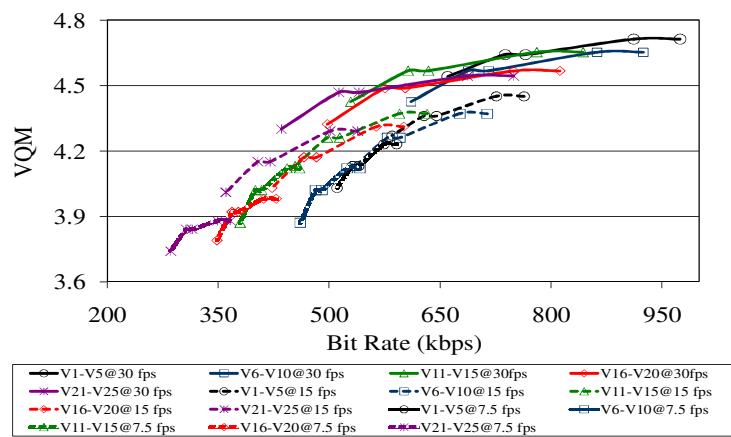

(c)

Fig. 8. VQM versus bit rate results for (a) Weather Forecast (b) Big Ships (c) B. Proposed Prioritization Schemes

In order to devise a prioritization scheme associated with a ML, the R-D performances of the points on the graphs of each adapted sequence are first analyzed. Secondly, NALUs associated with Vs (see Table V) and frame rates of the points are prioritized according to the results of the analysis and a list including the Vs and frame rates of the prioritized NALUs is prepared for each sequence. The three frame rates used to adapt the video sequences (i.e., 7.5, 15, and $30 \mathrm{fps}$ ) are associated with temporal layers 0,1 , and 2 , respectively, in the lists. It should be noted that in order to adapt a bit stream using higher temporal and quality enhancement layer NALUs, the lower temporal and quality enhancement layer NALUs should

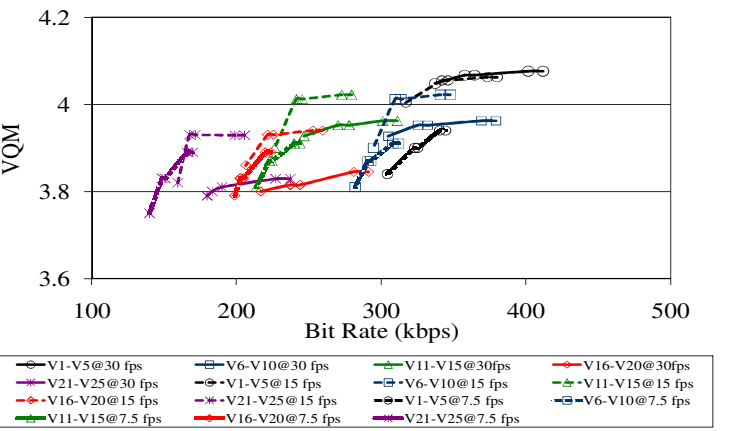

(b)

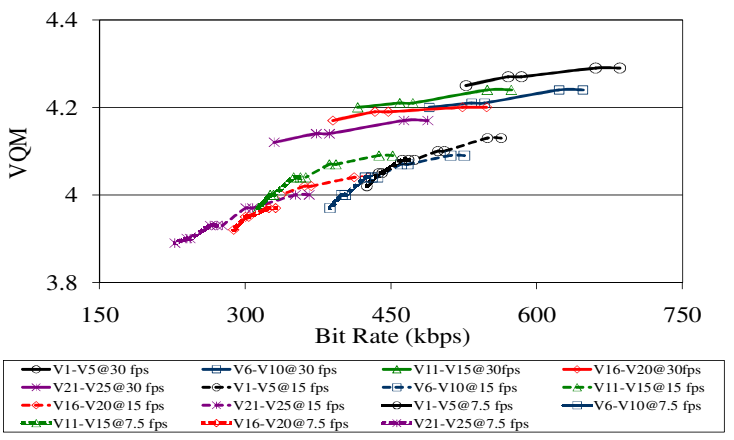

(d)

Tempete (d) Tennis sequences

be present in the bit stream. Hence, thirdly, starting from the NALU having the lowest priority, the most appropriate NALUs are selected from each list to determine new lists that can be used to devise prioritization schemes. It is observed from the new lists that the NALUs and their priorities are same for the sequences having similar MLs. Thus, a list of prioritized NALUs are associated with each ML (i.e., A, B, C, and D), as shown in Table VI. Lastly, each of these prioritized NALUs is analyzed to devise a generic prioritization scheme for each of the MLs. The flowchart of the devised prioritization schemes is shown in Fig. 9. 


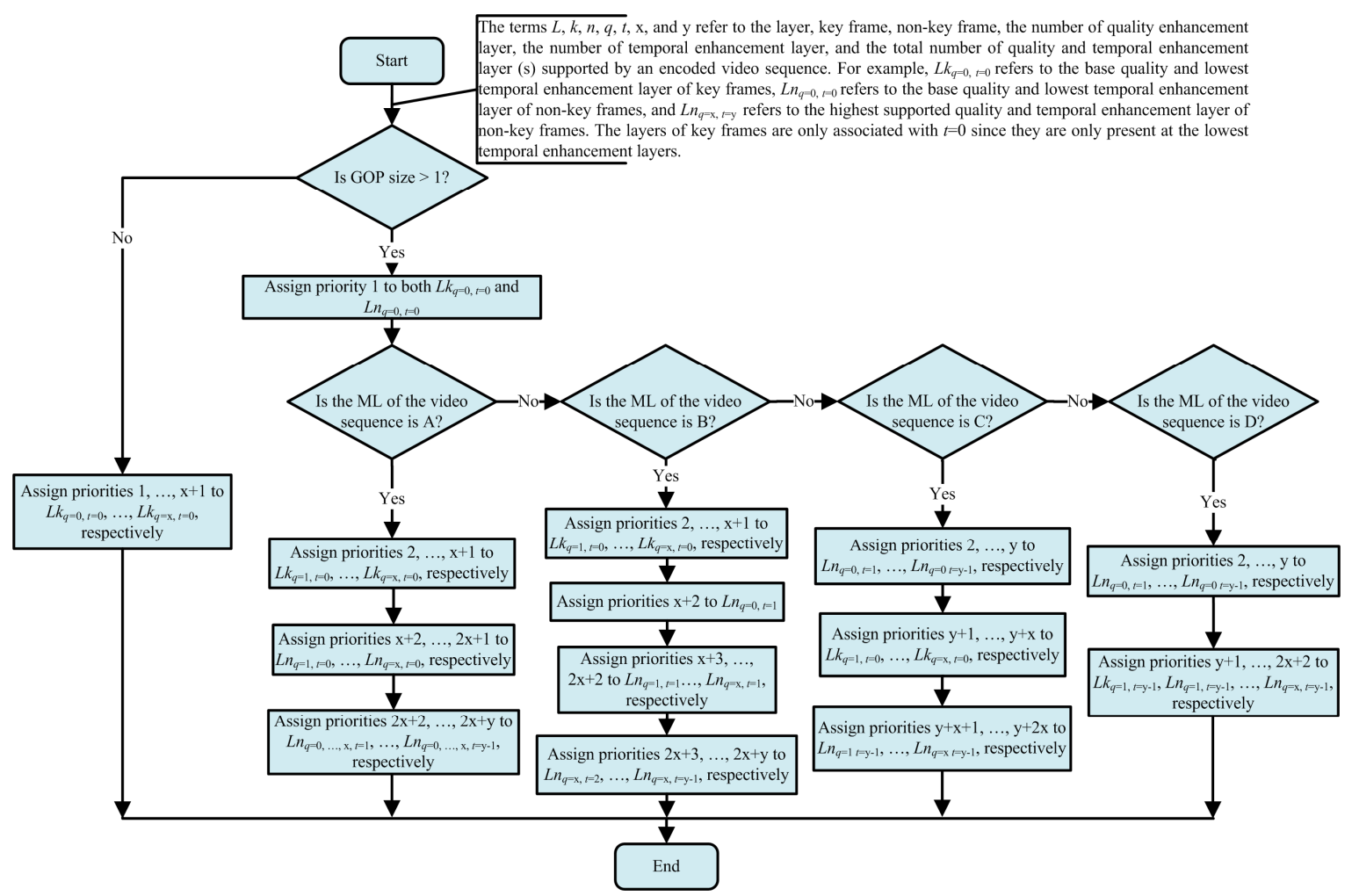

Fig. 9. The flow chart of the prioritization schemes for ML classes (a) A (b) B (c) C (d) D

TABLE VI

PRIORITIZED NALUS ASSOCIATED WITH KEY FRAME/NON-KEY FRAME/TEMPORAL LAYER

\begin{tabular}{|c|c|c|c|c|}
\hline \multirow{2}{*}{ Priority } & \multicolumn{4}{|c|}{ ML } \\
\cline { 2 - 5 } & $\mathrm{A}$ & $\mathrm{B}$ & $\mathrm{C}$ & $\mathrm{D}$ \\
\hline 1 & $0 / 0 / 0$ & $0 / 0 / 0$ & $0 / 0 / 0$ & $0 / 0 / 0$ \\
\hline 2 & $1 / 0 / 0$ & $1 / 0 / 0$ & $0 / 0 / 1$ & $0 / 0 / 1$ \\
\hline 3 & $2 / 0 / 0$ & $2 / 0 / 0$ & $0 / 0 / 2$ & $0 / 0 / 2$ \\
\hline 4 & $3 / 0 / 0$ & $3 / 0 / 0$ & $1 / 0 / 2$ & $1 / 0 / 2$ \\
\hline 5 & $4 / 0 / 0$ & $4 / 0 / 0$ & $2 / 0 / 2$ & $1 / 1 / 2$ \\
\hline 6 & $4 / 1 / 0$ & $4 / 0 / 1$ & $3 / 0 / 2$ & $2 / 1 / 2$ \\
\hline 7 & $4 / 2 / 0$ & $4 / 1 / 1$ & $4 / 0 / 2$ & $2 / 2 / 2$ \\
\hline 8 & $4 / 3 / 0$ & $4 / 2 / 1$ & $4 / 1 / 2$ & $3 / 2 / 2$ \\
\hline 9 & $4 / 4 / 0$ & $4 / 3 / 1$ & $4 / 2 / 2$ & $3 / 3 / 2$ \\
\hline 10 & $4 / 4 / 1$ & $4 / 4 / 1$ & $4 / 3 / 2$ & $4 / 3 / 2$ \\
\hline
\end{tabular}

\section{VIDEO BIT RATE ADAPTATION TECHNIQUE USING AMBIENT} ILLUMINATION CONTEXT

The sensitivity of Human Visual System (HVS) towards perceiving visual artifacts in a video sequence is associated with the amount of light the eyes capture from the viewing environment, and the iris' adaptation of its size according to the captured amount. When a video sequence is viewed in a dark room, most of the light is captured from the device used to view the sequence. The iris enlarges to let more light coming from the device in. As a result, the visual artifacts in the sequence become more visible to the eye.

When the video sequence is viewed in a bright room, the eyes capture the ambient light from the device, light bulbs, windows in the room, etc. Thus, the size of the iris decreases to allow less amount of light in and only a small amount of light is captured from the device. Therefore, the visual artifacts become less visible to the eye in a bright environment [27] [28]. The sensitivity of HVS towards detecting sharpness, shadows, reflections, contrast, etc in the visual content, all of which are essential cues to enhance depth perception in 3D video, decreases due to the increase in the ambient illumination [29] [30]. The sensitivity of the HVS towards perceiving overall quality of $3 \mathrm{D}$ video sequences viewed under different ambient illumination conditions corresponds to how the combination of video quality and depth is perceived. This fact is supported with the subjective experiments conducted under different ambient illumination conditions in this paper.

1) Experimental Set-up for the Subjective Assessments

Nine different test sequences namely; Butterfly, Couples, Ice, Windmill, Advertisement, Chess, Eagle, Football, and Interview were used in the subjective experiments. The test sequences were of High Definition (HD) resolution (i.e., $1920 \times 1080$ pixels) at 25 fps. The JSVM 9.13.1 [23] was used for scalable encoding of the test sequences using three quality enhancement layers. MGS [12] was used as the quality scalability support.

Four different channel bandwidths (i.e., 512, 768, 1024, and $1536 \mathrm{kbps}$ ) were selected as target bit rates. $80 \%$ of the target bit rate was allocated to the color sequences and the remaining bit rate (i.e., 20\%) was allocated to the depth map sequences. Different constant QP sets were used to encode the base and quality enhancement layers of color and depth map sequences to match the bit rates of the encoded video sequences to the target bit rates, and the best matching sets were used for the experiments. A 42"-Philips multi-view auto-stereoscopic display, which had a resolution of $1920 \times 1080$ pixels, was used to display the sequences in the experiments. 18 viewers (6 females and 12 males) participated in the experiments. After the outliers were screened using the outlier detection method introduced in [25] and removed, the MOS scores of 16 viewers ( 5 females and 11 males) were utilized for the experiments. 3D 
perceptual quality was assessed in four different ambient illumination conditions (i.e., 5, 52, 116, and 192 lux) during the experiments. These conditions were measured using a Gretag Macbeth Eye-One Display 2 device [32].

2) Subjective Assessment Results

The results of the subjective assessments for four selected 3D video sequences are shown in Fig. 10. As seen from the figures, the ambient illumination conditions in which the overall 3D perceptual quality is at the highest level vary for the sequences. The reason of the difference in the ambient illumination conditions in which the overall 3D video quality is perceived better is based on the presence of natural depth effect (e.g., due to relative distance of objects and background, relative size of objects in the scene, etc) in the color texture sequence. Moreover, according to the severity of natural depth effect, the relative importance of the video quality and depth perception related cues to enhance overall 3D perceptual quality changes under a particular ambient illumination condition. For instance, if the color texture of the 3D video sequence presents a high natural depth effect, the viewers tend to overlook ambient illumination's effect on depth perception but concentrate on its effect on video quality in a well-lit environment. Accordingly, the highest perceived video quality is achieved in this environment due to undistinguishable visual artifacts in the 3D video sequence, as shown in Fig. 10 (b).

As also observed, the points marked as A B, and C, which are on 5, 52, and 116 lux curves respectively, have similar MOSs versus different bit rates. Thus, when the ambient illumination of the viewing environment changes from low to high (e.g., from 5 to 52 or 5 to 116 or 52 to $116 \operatorname{lux}$ ), the overall $3 \mathrm{D}$ perceptual quality of an input sequence is not compromised by viewing it at a lower bit rate.

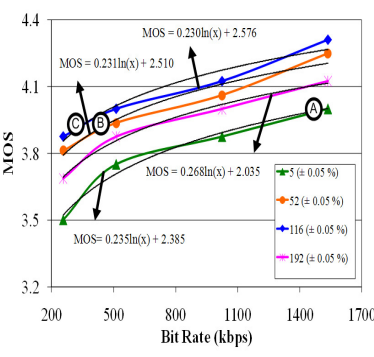

(a)

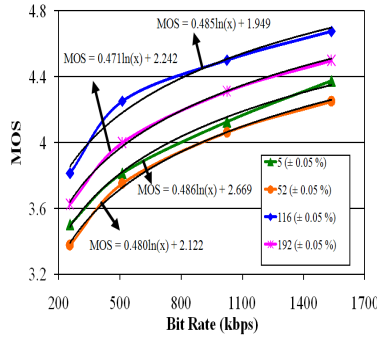

(c)

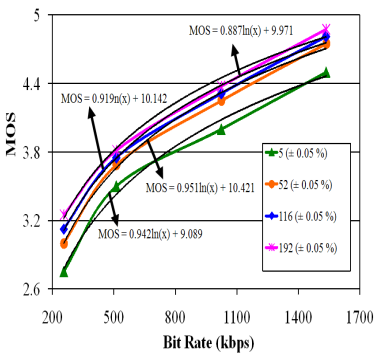

(b)

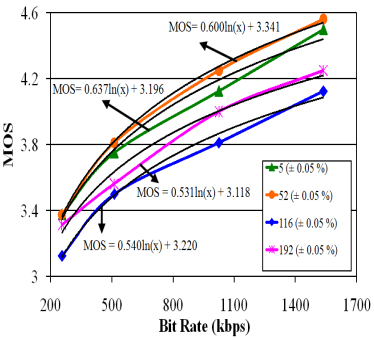

(d)
Fig. 10. The MOSs of initial experiments for (a) Butterfly (b) Chess (c) Eagle (d) Windmill sequences

\section{3) User Perception Model}

To model video perception of users considering different ambient illumination conditions, firstly, the mathematical functions of the curves in the graphs of subjective experiments results are determined. It is observed that the curves present the following pattern:

$$
M O S=W \ln (B)+Z
$$

where, $\mathrm{B}$ is bit rate and $W$ and $Z$ are constants. As the second step, the generic functions for $W$ and $Z$ are devised. In this way, MOS (or perceptual video quality) for a video sequence having $B$ bit rate can be calculated for an ambient illumination condition. For devising the generic functions of the constants (i.e., $W$ and $Z$ ), it is proposed to determine the contextual information affecting the values of the constants, and then find the correlation between the determined factors and constant values. For this purpose, the $W$ and $Z$ values of the curves in the graphs are presented in Table VII.

The $W$ and $Z$ values of the curves in Fig. 10 are shown in Table VII. As seen from the table, one of the contextual information influencing the constant values is ambient illumination, which is denoted by $I$, since when the $I$ changes, the values of the constants also vary. As can also be realized from the table, the values of the constants are also content dependent for the same $I$. The content related contextual information affecting the values of the constants are discussed in the following sub-section.

TABLE VII

EXPERIMENTAL AND PREDICTED VALUES OF $W$ AND $Z$ USING THEIR GENERIC

\begin{tabular}{|c|c|c|c|c|c|c|c|c|c|}
\hline & \multirow{3}{*}{$M$} & & \multirow{3}{*}{$L$} & \multirow{3}{*}{$D$} & \multirow{3}{*}{$I$} & CTION & & & \\
\hline \multirow{2}{*}{ Seq. } & & \multirow{2}{*}{ C } & & & & \multicolumn{2}{|c|}{ Experimental } & \multicolumn{2}{|c|}{ Predicted } \\
\hline & & & & & & $W$ & $Z$ & $W$ & $Z$ \\
\hline \multirow{4}{*}{ 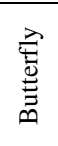 } & \multirow{4}{*}{ 三 } & \multirow{4}{*}{$\begin{array}{l}\text { త్ } \\
\text { o. }\end{array}$} & \multirow{4}{*}{$\begin{array}{l}\text { ণ } \\
\text { ণ }\end{array}$} & \multirow{4}{*}{$\begin{array}{l}\stackrel{n}{f} \\
\stackrel{0}{0} \\
\stackrel{\overbrace{}}{\sim}\end{array}$} & 5 & 0.235 & 2.385 & 0.274 & 1.403 \\
\hline & & & & & 52 & 0.231 & 2.510 & 0.237 & 1.614 \\
\hline & & & & & 116 & 0.230 & 2.576 & 0.242 & 1.603 \\
\hline & & & & & 192 & 0.268 & 2.035 & 0.243 & 1.585 \\
\hline \multirow{4}{*}{$\stackrel{\tilde{d}}{\stackrel{\tilde{v}}{U}}$} & \multirow{4}{*}{$\frac{N}{?}$} & \multirow{4}{*}{$\frac{\vec{J}}{\dot{0}}$} & \multirow{4}{*}{$\begin{array}{l}\stackrel{8}{8} \\
\stackrel{+}{+}\end{array}$} & \multirow{4}{*}{$\frac{m}{\stackrel{0}{\sigma}}$} & 5 & 0.942 & 9.089 & 1.371 & 8.926 \\
\hline & & & & & 52 & 0.951 & 10.421 & 1.229 & 10.275 \\
\hline & & & & & 116 & 0.919 & 10.142 & 1.394 & 10.099 \\
\hline & & & & & 192 & 0.887 & 9.971 & 1.211 & 10.085 \\
\hline \multirow{4}{*}{ 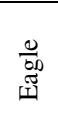 } & \multirow{4}{*}{ స̂. } & \multirow{4}{*}{$\begin{array}{c}\tilde{m} \\
0 \\
0 \\
0\end{array}$} & \multirow{4}{*}{$\frac{\vec{d}}{\vec{g}}$} & \multirow{4}{*}{$\begin{array}{l}\text { J̦ } \\
\text { م̂} \\
\stackrel{n}{n}\end{array}$} & 5 & 0.486 & 2.669 & 1.477 & 2.807 \\
\hline & & & & & 52 & 0.480 & 2.122 & 1.365 & 3.232 \\
\hline & & & & & 116 & 0.485 & 1.949 & 1.438 & 3.208 \\
\hline & & & & & 192 & 0.471 & 2.242 & 1.393 & 3.172 \\
\hline \multirow{4}{*}{ 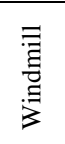 } & \multirow{4}{*}{$\left|\begin{array}{c}2 \\
\infty \\
0\end{array}\right|$} & \multirow{4}{*}{$\frac{\grave{\beth}}{\stackrel{0}{0}}$} & \multirow{4}{*}{$\begin{array}{l}\infty \\
\stackrel{8}{ } \\
\infty \\
\text { in }\end{array}$} & \multirow{4}{*}{ 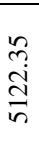 } & 5 & 0.637 & 3.096 & 0.516 & 0.373 \\
\hline & & & & & 52 & 0.600 & 3.341 & 0.515 & 0.429 \\
\hline & & & & & 116 & 0.540 & 3.220 & 0.486 & 0.427 \\
\hline & & & & & 192 & 0.531 & 3.118 & 0.490 & 0.421 \\
\hline
\end{tabular}

a) Content Related Contextual Information of the User Perception Model

It is established that motion activity and structural feature characteristics of video contents are video quality perception related contextual information influencing the values of the constants. Therefore, the metrics proposed in sub-section II/A/1 and II/A/2 are used as the content related contextual information to calculate the values of the constants. Content related contextual information associated with depth perception has also influence on $3 \mathrm{D}$ video quality. Thus, luminance contrast and depth intensity, which affect depth perception, are also used to determine the values of the constants.

\section{(1) Luminance Contrast}

The luminance contrast of a color texture frame is measured using Median Absolute Deviation (MAD) [32] in this paper. The $M A D$ s computed per frame are then integrated together to determine the $M A D$ across a color texture sequence. The 
calculated $M A D$ s are then normalized with $\mathrm{NoF}$ and $S$ to provide consistent measurement among different video sequences. Thus, the metric below is devised for luminance contrast measurements:

$$
L=\frac{\sum_{i=1}^{\mathrm{NoF}} M A D(i)}{\operatorname{NoF} S}
$$

where, $M A D(i)$ is the luminance contrast of the $i^{\text {th }}$ frame of a color texture sequence, $L$ is the luminance contrast of the color texture sequence.

$$
\text { (2) Depth Intensity }
$$

Depth intensity of a depth map frame is measured by applying standard deviation [15] to each frame. Then, the standard deviation values calculated per frame are integrated together to measure the depth intensity across a depth map, as presented below:

$$
D=\frac{\sum_{i=1}^{\mathrm{NoF}} S D(i)}{\mathrm{NoF}}
$$

where, $S D(i)$ is the standard deviation of the $i^{\text {th }}$ frame of a depth map, $D$ is the depth intensity of the depth map.

\section{b) Generic Functions for the Constants}

The following functions are obtained to devise generic functions for $W$ and $Z$ after approximating $M, C, L$, and $D$, with $W$ and $Z$ using curve fitting functions [33], respectively, and introducing a set of constants to each of the approximation function:

$$
\begin{gathered}
W \propto h(M)=(M+0.15) \\
W \propto h(C)=\left(\frac{C^{2}+1}{15}\right) \\
W \propto h(L)=\left(\frac{L+0.01}{\ln \left(L^{2}+1\right)}\right) \\
W \propto h(D)=\left(\frac{10^{3}(D+100)}{D^{2}+1}\right) \\
W \propto h(I)=\left(\frac{e^{\sqrt{\ln (I+1)}}}{\ln (I+1)}\right) \\
Z \propto p(M)=\left(\frac{M^{2}+2 M+0.5}{M+0.7}\right) \\
Z \propto p(C)=\left(\frac{C^{5}+3 C^{3}+5 C^{2}+C+1}{2 C^{3}+C^{2}+3 C+1}\right) \\
Z \propto p(L)=\left(\frac{2(L+0.5)}{\left(\ln \left(L^{2}+1\right)\right)^{2}}\right) \\
Z \propto p(D)=\left(\left(\frac{D^{3}+2 D^{2}+D+0.1}{D^{2}+3 D+2}\right)+0.5\right) \\
Z \propto p(I)=\left(\left(\frac{\ln (I+1)+0.01}{\left.e^{\sqrt{\ln (I+1)}}\right) 10^{-4}}\right)\right.
\end{gathered}
$$

Subsequently, the functions of $W$ and $Z$ are integrated together to devise generic functions as follows:

$$
W=(h(M) h(C) h(D) h(L) h(I)), Z=(p(M) p(C) p(D) p(L) p(I))
$$

4) Proposed Adaptation Decision Taking Technique

Taking into consideration that the perceptual qualities for input and adapted video sequences should be maintained regardless of the difference in the ambient illumination of the viewing environment, an assumption can be made, which is described as follows:

$$
W_{\text {input }} \ln \left(B_{\text {input }}\right)+Z_{\text {input }}=W_{\text {adaped }} \ln \left(B_{\text {adapted }}\right)+Z_{\text {adapted }}
$$

$$
B_{\text {adapted }}=e^{\frac{W_{\text {input }} \ln \left(B_{\text {input }}\right)+Z_{\text {ipput }}-Z_{\text {adapted }}}{W_{\text {adapted }}}}
$$

where, $W_{\text {input }}$ and $Z_{\text {input }}$ are the constants for input 3D video sequence and $W_{\text {adapted }}$ and $Z_{\text {adapted }}$ are the constants for the adapted 3D video sequence. (13) can be used to compute the bit rate for adapting an input $3 \mathrm{D}$ video sequence $\left(B_{\text {adapted }}\right)$ from a given input bit rate $\left(B_{\text {input }}\right)$ under a specific amount of ambient illumination change while maintaining the perceived $3 \mathrm{D}$ video quality.

\section{RESULTS AND DISCUSSION}

A. Utility-based Scalable Video Adaptation Technique Using Spatial, Temporal, and Quality Scalability Parameters

Adaptation decision taking and adaptation experiments were conducted using the proposed technique and the study defined in [2] as a reference technique in this paper. The study discussed in [2] is based on maximizing bit rate of video sequences to determine the adaptation parameters. Moreover, content related contextual information is not considered in the study discussed in [2].

For the experiments, test sequences namely: New, Sheriff, Coastguard, Football, Silent, Big Ships, Flower Garden, and Tennis were encoded with three spatial layers (i.e., QCIF, CIF, and SD), four temporal layers (i.e., 3.75, 7.5, 15, and $30 \mathrm{fps}$ ), and two MGS layers for each spatial layer using JSVM 9.13.1 [23]. The sequences belong to the ML classes 1-8, respectively (see Table III). The target network bandwidths $=\{200,500$, 700 , and 1000$\} \mathrm{kbps}$, display width $=704$ pixels, and display height $=576$ pixels were applied as constraints to restrict adaptation decisions of the proposed and reference adaptation techniques. Then, sub-streams that have bit rates smaller than each of the target network bandwidths are extracted from the encoded test sequences. The parameters of these sub-streams are called as candidate adaptation parameters. The parameters that maximize the proposed UF (see (1)) are then selected among the candidate parameters for each target network bandwidth. These parameters refer to the adaptation decision taking results of the proposed technique. The parameters of the sub-streams that have the maximum bit rates among the candidate adaptation parameters refer to the adaptation decision taking results of the reference technique. The adaptation decision taking results of the proposed and reference techniques are illustrated in Table VIII.

Using the adaptation decision taking results, adaptation experiments were conducted, and the adapted test sequences were evaluated with subjective experiments. DSIS [24] was employed for the subjective tests. 18 viewers participated to the experiments. After the outliers were detected, using the outlier screening method in [25], and removed, 16 observers were used to calculate the MOSs in the experiments. The MOSs of the subjective experiments are shown together with the confidence intervals on the right hand side of the table. As observed from The MOSs, the proposed technique outperforms the reference technique for most of the adapted sequences. 
B. Video Adaptation technique Using Symmetric and Asymmetric Key/Non-key Frame Quality Scalability Ordering Based Prioritization

Adaptation experiments were conducted using the proposed and conventional adaptation techniques to assess the adaptation performance of the proposed technique. The Hall Monitor, Carphone, City, and Soccer sequences, which have MLs of A, B, C, and D, respectively (see Table I), were exploited in the experiments. These sequences were encoded with one base layer (level 0), and, two MGS stacks each having two quality enhancement layers (i.e., four quality enhancement layers in total (levels 1, 2, 3, and 4)) with a GOP size of 4, using the JSVM 9.13.1 codec [23]. For the experiments, the NALUs of the encoded sequences were prioritized using the corresponding proposed prioritization schemes (see Fig. 9). After the NALUs were prioritized, the video sequences were adapted considering different network bandwidths. Then, the VQM grades of the adapted sequences were computed. The results of the adaptation experiments carried out using the proposed prioritization schemes are presented in Table IX.

The NALUs of the encoded sequences were also prioritized according to the following variations of Table II: V1 at 7.5, 15 , and $30 \mathrm{fps}$, and V7, V13, V19, and V25 at $30 \mathrm{fps}$, which correspond to the conventional prioritization order. The conventional prioritization order is used as the reference technique to compare the R-D performance of the proposed prioritization schemes.

After the NALUs were prioritized using this order, the video sequences were adapted based on the same network bandwidths. Afterwards, the VQM grades of the adapted sequences were calculated. Table IX also illustrates the results of the adaptation experiments conducted considering conventional prioritization order. As can be observed from the table, the results of the proposed prioritization scheme outperform those of the conventional one in terms of either VQM grades or lower bit rates. Thus, the R-D performances of adapted video sequences can be improved using the proposed prioritization schemes.

TABLE VIII

COMPARISON OF THE ADAPTATION DECISION TAKING RESUlTS OF THE PROPOSED (PROP.) AND REFERENCE (REF.) TECHNIQUES FOR DIFFERENT SEQUENCES

\begin{tabular}{|c|c|c|c|c|c|c|c|c|c|c|c|c|}
\hline \multirow{2}{*}{ ڤ̆ } & \multirow{2}{*}{$\begin{array}{c}\text { Network } \\
\text { Bandwidth } \\
\text { (kbps) }\end{array}$} & \multicolumn{2}{|c|}{$\begin{array}{c}\text { Bit Rate } \\
(\mathrm{kbps})\end{array}$} & \multicolumn{2}{|c|}{$\begin{array}{c}\text { Spatial } \\
\text { Resolution }\end{array}$} & \multicolumn{2}{|c|}{$\begin{array}{c}\text { Frame Rate } \\
(\mathrm{kbps})\end{array}$} & \multirow[t]{2}{*}{ VQM } & \multicolumn{2}{|c|}{ SNR } & \multicolumn{2}{|c|}{ MOS \pm Confidence Interval } \\
\hline & & prop. & ref. & prop. & ref. & prop. & ref. & & prop. & ref. & prop. & ref. \\
\hline \multirow{4}{*}{$\mid \begin{array}{l}n \\
\vdots \\
0 \\
z \\
z\end{array}$} & 200 & 179 & 199 & $\mathrm{CIF}$ & CIF & 7.5 & 3.75 & 4.21 & 1 & 2 & $4.12 \pm 0.31$ & $3.93 \pm 0.42$ \\
\hline & 500 & 499 & 499 & SD & SD & 7.5 & 7.5 & 4.11 & 0 & 0 & $4.25 \pm 0.35$ & $4.25 \pm 0.35$ \\
\hline & 700 & 551 & 632 & SD & SD & 7.5 & 15 & 4.14 & 1 & 0 & $4.44 \pm 0.31$ & $4.31 \pm 0.29$ \\
\hline & 1000 & 932 & 932 & SD & SD & 7.5 & 7.5 & 4.57 & 2 & 2 & $4.56 \pm 0.24$ & $4.56 \pm 0.24$ \\
\hline \multirow{4}{*}{ 言 } & 200 & 192 & 192 & QCIF & QCIF & 7.5 & 7.5 & 4.49 & 1 & 1 & $3.56 \pm 0.37$ & $3.56 \pm 0.37$ \\
\hline & 500 & 467 & 498 & $\mathrm{CIF}$ & $\overline{\mathrm{QCIF}}$ & 7.5 & 15 & 4.53 & 1 & 2 & $3.87 \pm 0.30$ & $3.50 \pm 0.31$ \\
\hline & 700 & 584 & 699 & $\mathrm{CIF}$ & QCIF & 7.5 & 30 & 4.90 & 2 & 1 & $3.93 \pm 0.42$ & $3.75 \pm 0.33$ \\
\hline & 1000 & 965 & 998 & SD & SD & 7.5 & 3.75 & 4.82 & 1 & 2 & $4.12 \pm 0.42$ & $4.06 \pm 0.34$ \\
\hline \multirow{4}{*}{ ن. } & 200 & 158 & 158 & QCIF & QCIF & 15 & 15 & 3.96 & 2 & 2 & $2.94 \pm 0.33$ & $2.94 \pm 0.33$ \\
\hline & 500 & 452 & 492 & $\mathrm{CIF}$ & $\mathrm{CIF}$ & 7.5 & 15 & 3.18 & 0 & 1 & $3.12 \pm 0.30$ & $3.06 \pm 0.35$ \\
\hline & 700 & 575 & 642 & $\mathrm{CIF}$ & $\mathrm{CIF}$ & 30 & 7.5 & 3.16 & 0 & 1 & $3.31 \pm 0.29$ & $3.19 \pm 0.27$ \\
\hline & 1000 & 911 & 999 & $\mathrm{CIF}$ & SD & 30 & 3.75 & 3.64 & 1 & 0 & $3.50 \pm 0.25$ & $3.06 \pm 0.22$ \\
\hline \multirow{4}{*}{. } & 200 & 191 & 191 & QCIF & QCIF & 15 & 15 & 3.53 & 0 & 0 & $2.81 \pm 0.27$ & $2.81 \pm 0.27$ \\
\hline & 500 & 404 & 497 & QCIF & CIF & 30 & 3.75 & 3.95 & 2 & 0 & $3.31 \pm 0.29$ & $2.69 \pm 0.34$ \\
\hline & 700 & 513 & 623 & QCIF & $\mathrm{CIF}$ & 30 & 7.5 & 3.95 & 2 & 0 & $3.37 \pm 0.29$ & $3.06 \pm 0.22$ \\
\hline & 1000 & 908 & 943 & $\mathrm{CIF}$ & $\mathrm{CIF}$ & 15 & 7.5 & 3.49 & 0 & 2 & $3.62 \pm 0.35$ & $3.43 \pm 0.40$ \\
\hline \multirow{4}{*}{ 离 } & 200 & 189 & 189 & $\mathrm{CIF}$ & CIF & 7.5 & 7.5 & 3.47 & 0 & 0 & $3.56 \pm 0.31$ & $3.56 \pm 0.31$ \\
\hline & 500 & 430 & 431 & $\mathrm{CIF}$ & $\mathrm{CIF}$ & 7.5 & 30 & 3.93 & 2 & 1 & $3.87 \pm 0.43$ & $3.48 \pm 0.48$ \\
\hline & 700 & 648 & 648 & SD & SD & 7.5 & 7.5 & 3.98 & 1 & 1 & $3.91 \pm 0.38$ & $3.91 \pm 0.38$ \\
\hline & 1000 & 806 & 972 & SD & SD & 15 & 7.5 & 3.88 & 1 & 2 & $4.12 \pm 0.39$ & $4.06 \pm 0.44$ \\
\hline \multirow{4}{*}{$\begin{array}{l}\stackrel{2}{3} \\
\tilde{\omega} \\
\cdots \infty \\
\cdots\end{array}$} & 200 & 154 & 162 & $\mathrm{CIF}$ & CIF & 15 & 30 & 3.17 & 0 & 0 & $3.43 \pm 0.44$ & $3.43 \pm 0.44$ \\
\hline & 500 & 383 & 396 & $\mathrm{CIF}$ & $\mathrm{CIF}$ & 15 & 30 & 4.35 & 2 & 2 & $3.62 \pm 0.39$ & $3.56 \pm 0.39$ \\
\hline & 700 & 687 & 687 & SD & SD & 15 & 15 & 4.12 & 1 & 1 & $3.93 \pm 0.45$ & $3.93 \pm 0.46$ \\
\hline & 1000 & 929 & 992 & SD & SD & 7.5 & 15 & 4.78 & 2 & 2 & $4.37 \pm 0.42$ & $4.25 \pm 0.31$ \\
\hline \multirow{4}{*}{ 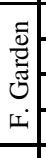 } & 200 & 152 & 191 & QCIF & QCIF & 30 & 15 & 4.25 & 1 & 2 & $2.81 \pm 0.37$ & $2.68 \pm 0.38$ \\
\hline & 500 & 220 & 454 & QCIF & CIF & 30 & 3.75 & 4.56 & 2 & 0 & $3.18 \pm 0.30$ & $3.00 \pm 0.43$ \\
\hline & 700 & 640 & 677 & $\mathrm{CIF}$ & $\mathrm{CIF}$ & 15 & 7.5 & 3.67 & 0 & 1 & $3.56 \pm 0.39$ & $3.31 \pm 0.35$ \\
\hline & 1000 & 963 & 963 & $\mathrm{CIF}$ & CIF & 30 & 30 & 4.24 & 1 & 1 & $3.93 \pm 0.33$ & $3.93 \pm 0.33$ \\
\hline \multirow{4}{*}{ 迹 } & 200 & 192 & 192 & QCIF & QCIF & 30 & 30 & 4.10 & 2 & 2 & $3.25 \pm 0.38$ & $3.25 \pm 0.38$ \\
\hline & 500 & 415 & 494 & $\mathrm{CIF}$ & CIF & 15 & 3.75 & 3.64 & 0 & 1 & $3.37 \pm 0.47$ & $2.75 \pm 0.38$ \\
\hline & 700 & 507 & 638 & $\mathrm{CIF}$ & CIF & 30 & 7.5 & 3.70 & 0 & 2 & $3.68 \pm 0.44$ & $3.50 \pm 0.25$ \\
\hline & 1000 & 968 & 968 & $\mathrm{CIF}$ & $\mathrm{CIF}$ & 30 & 30 & 4.24 & 2 & 2 & $4.06 \pm 0.53$ & $4.06 \pm 0.53$ \\
\hline
\end{tabular}

C. Video Bit Rate Adaptation Technique Using Ambient Illumination Context

In order to validate the efficiency of the proposed technique, adaptation decision taking experiments were conducted using (13). Moreover, adaptation experiments were performed considering the outcomes of the adaptation decision taking experiments. It should be noted that the computational complexity of the proposed technique solely depend on the calculation of the (13), which is low. Ambient illumination condition in which the input 3D video sequences is viewed is 
kept as 5 lux to maintain common reference ambient illumination conditions for the experiments.

However, five different ambient illumination conditions ( $\left.I_{\text {adapted }}\right)$ were set to adapt $3 \mathrm{D}$ video sequences for the experiments. The experiments were carried out for 3D input sequences having three different input bit rates (i.e., $B_{\text {input }}=$ 384, 512, and $1024 \mathrm{kbps})$. A 42"-Philips multi-view autostereoscopic display, which has a resolution of $1920 \times 1080$ pixels, was used to display the $3 \mathrm{D}$ sequences in the experiments. The 3D video sequences fill up the device displays in the experiments. Table $\mathrm{X}$ presents the resultant adapted bit rates $\left(B_{\text {adapted }}\right)$ for the $3 \mathrm{D}$ input sequences.

To validate the resulting $B_{\text {adapted }}$ s for the adapted sequences, further subjective experiments were conducted. The adapted

TABLE IX

EXPERIMENTAL RESULTS COMPRISED USING THE PROPOSED SCHEME AND CONVENTIONAL PRIORITIZATION ORDER

\begin{tabular}{|c|c|c|c|c|c|c|c|}
\hline \multirow[b]{2}{*}{$\dot{\bar{D}}$} & \multirow{2}{*}{$\begin{array}{c}\text { Network } \\
\text { Bandwidth } \\
\text { (kbps) }\end{array}$} & \multicolumn{3}{|c|}{ Proposed Prioritization Scheme } & \multicolumn{3}{|c|}{ Conventional Prioritization Order } \\
\hline & & $\begin{array}{c}\text { Bit Rate } \\
\text { (kbps) }\end{array}$ & VQM & $\begin{array}{c}\text { Core Adaptation Parameters } \\
\text { (key frame/non-key frame/temporal layer) }\end{array}$ & $\begin{array}{c}\text { Bit Rate } \\
\text { (kbps) }\end{array}$ & VQM & $\begin{array}{c}\text { Core Adaptation Parameters } \\
\text { (key frame/non-key frame/temporal layer) }\end{array}$ \\
\hline \multirow{4}{*}{ 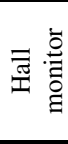 } & 250 & 238.24 & 4.01 & $2 / 0 / 0$ & 261.70 & 3.93 & $0 / 0 / 1$ \\
\hline & 450 & 436.41 & 4.26 & $4 / 4 / 0$ & 427.15 & 3.92 & $0 / 0 / 2$ \\
\hline & 650 & 586.58 & 4.33 & $4 / 4 / 1$ & 633.07 & 4.04 & $2 / 2 / 2$ \\
\hline & 1000 & 993.34 & 4.31 & $4 / 4 / 2$ & 993.34 & 4.31 & $4 / 4 / 2$ \\
\hline \multirow{3}{*}{$\frac{\mathscr{E}}{\frac{0}{2}}$} & 150 & 139.84 & 3.76 & $0 / 0 / 0$ & 142.76 & 3.77 & $0 / 0 / 2$ \\
\hline & 250 & 213.75 & 3.88 & $2 / 0 / 0$ & 243.84 & 3.92 & $2 / 2 / 2$ \\
\hline & 400 & 373.29 & 4.08 & $4 / 3 / 1$ & 359.04 & 3.94 & $3 / 3 / 2$ \\
\hline \multirow{4}{*}{$\underset{己}{己}$} & 300 & 258.89 & 3.89 & $0 / 0 / 1$ & 258.89 & 3.89 & $0 / 0 / 1$ \\
\hline & 500 & 475.71 & 4.05 & $1 / 0 / 2$ & 498.34 & 4.11 & $1 / 1 / 2$ \\
\hline & 600 & 520.98 & 4.08 & $2 / 0 / 2$ & 585.86 & 4.15 & $2 / 2 / 2$ \\
\hline & 850 & 801.09 & 4.22 & $4 / 2 / 2$ & 844.19 & 4.28 & $3 / 3 / 2$ \\
\hline \multirow{3}{*}{$\begin{array}{l}\bar{\delta} \\
\text { O } \\
\dot{\sim}\end{array}$} & 500 & 450.65 & 3.85 & $0 / 0 / 1$ & 450.65 & 3.85 & $0 / 0 / 1$ \\
\hline & 1000 & 948.12 & 4.47 & $2 / 1 / 2$ & 906.13 & 4.25 & $1 / 1 / 2$ \\
\hline & 1350 & 1304.59 & 4.68 & $4 / 3 / 2$ & 1283.09 & 4.51 & $3 / 3 / 2$ \\
\hline
\end{tabular}

TABLE X

RESULTING ADAPTED BIT RATES USING THE 3D VIDEO BIT RATE ADAPTATION TECHNIQUE

\begin{tabular}{|c|c|c|c|c|}
\hline \multirow{4}{*}{ Sequence } & \multirow{2}{*}{$\begin{array}{c}\text { adapted } \\
\text { (lux) }\end{array}$} & $\begin{array}{c}B_{\text {input }}=384- \\
\text { MOS }\end{array}$ & $\begin{array}{c}B_{\text {input }}=512- \\
\text { MOS }\end{array}$ & $\begin{array}{c}B_{\text {input }}=1024- \\
\text { MOS }\end{array}$ \\
\hline \multirow{5}{*}{ Butterfly } & 28 & $385.68-4.25$ & $531.45-4.43$ & $1172.47-4.56$ \\
\cline { 2 - 5 } & 52 & $398.91-4.25$ & $556.29-4.43$ & $1239.63-4.56$ \\
\cline { 2 - 5 } & 116 & $368.87-4.18$ & $510.88-4.37$ & $1119.74-4.50$ \\
\cline { 2 - 5 } & 164 & $417.43-4.25$ & $576.59-4.43$ & $1255.67-4.56$ \\
\cline { 2 - 5 } & 192 & $387.69-4.25$ & $536.21-4.43$ & $1171.49-4.56$ \\
\hline \multirow{5}{*}{ Chess } & 28 & $323.48-3.75$ & $450.84-3.87$ & $1003.19-4.31$ \\
\cline { 2 - 5 } & 52 & $254.67-3.75$ & $351.02-3.87$ & $760.53-4.25$ \\
\hline & 116 & $249.98-3.75$ & $299.01-3.87$ & $693.48-4.25$ \\
\cline { 2 - 5 } & 164 & $354.52-3.75$ & $490.07-3.87$ & $1019.22-4.25$ \\
\cline { 2 - 5 } & 192 & $323.49-3.75$ & $448.03-3.87$ & $981.91-4.31$ \\
\hline \multirow{5}{*}{ Eagle } & 28 & $457.89-4.00$ & $624.66-4.06$ & $1320.15-4.37$ \\
\cline { 2 - 5 } & 52 & $458.02-4.00$ & $625.26-4.06$ & $1323.59-4.31$ \\
\cline { 2 - 5 } & 116 & $341.23-3.87$ & $458.53-4.00$ & $934.39-4.31$ \\
\cline { 2 - 5 } & 164 & $417.23-4.00$ & $564.67-4.06$ & $1170.69-4.37$ \\
\cline { 2 - 5 } & 192 & $422.77-4.00$ & $573.54-4.06$ & $1195.94-4.37$ \\
\hline \multirow{5}{*}{ Windmill } & 28 & $496.84-4.18$ & $674.30-4.25$ & $1407.45-4.43$ \\
\hline & 52 & 348.234 .18 & $464.55-4.18$ & $930.28-4.43$ \\
\cline { 2 - 5 } & 116 & $495.82-4.06$ & $672.91-4.25$ & $1404.56-4.43$ \\
\hline & 164 & $549.38-4.18$ & $748.46-4.25$ & $1576.67-4.43$ \\
\hline & 192 & $477.13-4.18$ & $645.94-4.25$ & $1340.18-4.50$ \\
\hline
\end{tabular}

sequences were presented to the viewers under the five $I_{\text {adapted }}$ used in the experiments. The DSIS method [24] was used in the experiments. 18 viewers participated for the experiments, and after the outliers were detected, the MOS scores were calculated for 16 subjects in the experiments. The MOS results of the 3D subjective assessments can also be seen from Table $X$. As can be observed from the results, the perceptual qualities of the adapted 3D video sequences slightly vary $(\sim 0.02 \%)$ under changing ambient illumination conditions. Accordingly, it can be argued that the proposed techniques have been efficient to adapt the $3 \mathrm{D}$ video sequences while maintaining their respective perceptual qualities despite the different viewing ambient illumination_conditions.

\section{:}


[5] S. F. Chang, "Optimal Video Adaptation and Skimming Using a UtilityBased Framework,"Int. W. on Dig. Com., Capri Island, Italy, Sep. 2002.

[6] M. Prangl, T. Szkaliczki, H. Hellwagner, "A Framework for UtilityBased Multimedia Adaptation," IEEE TCSVT, vol. 17, no. 6, pp. 719728, Jun. 2007.

[7] O. D. Onur and A. A. Alatan, "Video Adaptation Based on Content Characteristics and Hardware Capabilities," Second Int. Workshop on Semantic Media Adap. and Personal., pp. 15-20, Uxbridge, 17-18 Dec. 2007.

[8] O. D. Onur and A. A. Alatan, "Optimal Video Adaptation for Resource Constrained Mobile Devices Based on Utility Theory," Workshop on Image Analysis for Multimedia Int. Services, Portugal, 2004.

[9] O. D. Onur and A. A. Alatan, "Video Adaptation for Transmission Channels by Utility Modeling," IEEE Int. Conf. on Mult. and Expo, Amsterdam, The Netherlands, 6 Jul. 2005.

[10] A. A. Sofokleous and M. C. Angelides, "DCAF: An MPEG-21 Dynamic Content Adaptation Framework," Springer Multimedia Tools Applications, vol. 40, pp. 151-182, 2008.

[11] I. Amonou, N. Cammas, S. Kervadec, and S. Pateux, "Optimized RateDistortion Extraction with Quality Layers in the Scalable Extension of H.264/AVC," IEEE TCSVT, vol. 17, pp. 1186-1193, Sep. 2007.

[12] T. C. Thang, J.-G. Kim, J. W. Kang, and J.-J. Yoo, "SVC Adaptation: Standard Tools and Supporting Methods," Elsevier Signal Proc.: Image Com., vol. 24, pp. 214-228, 2009.

[13] G. Nur, H. Kodikara Arachchi, S. Dogan, and A. M. Kondoz, "A Novel Scalability Adaptation Concept for Higher R-D Performances of Scalable Video", ICT Mobile and Wireless Com. Summit, Santander, Spain, 10-12 Jun. 2009.

[14] I. Kofler, C. Timmerer, H. Hellwagner, A. Hutter, F. Sanahuja, "Efficient MPEG-21 Based Adaptation Decision Taking for Scalable Multimedia Content," Proc. of the 14th SPIE Annual Elect. Imaging Conf.-Mult. Comp. and Networking, San Jose, CA, USA, 31 Jan. 2007.

[15] J. L. Devore, "Probability and Statistics for Engineering and the Sciences", $4^{\text {th }}$ edition, pp. 474-585, Duxbury, 1995.

[16] M.H. Pinson and S .Wolf, "A New Standardized Method for Objectively Measuring Video Quality," IEEE Trans. on Broadcasting, vol. 50, no.3, pp. 312-322, Sep. 2004.

[17] J. Bouguet, "Pyramidal Implementation of the Lucas-Kanade Feature Tracker: Description of the Algorithm," Technical report, OpenCV Document, Intel Microprocessor Research Labs, 2000.

[18] J. Shi and C. Tomasi, "Good Features to Track," IEEE Conference on Computer Vision and Pattern Recognition, Seattle, USA, Jun. 1994.

[19] Q. Zhu, S. Avidan, and K. Cheng, "Learning a Sparse, Corner Based Representation for Time-varying Background Modelling," Proc. of the 10th Int. Conf. on Comp. Vision, Beijing, China, 17-21 Oct. 2005.

[20] M. H. Hsiao, Y. W. Chen, H. T. Chen, K. H. Chou, and S. Y. Lee, "Content-Aware Video Adaptation under Low-Bitrate Constraint,"
EURASIP Journal on Advances in Sig. Processing, vol. 2007, Article ID 17179, May. 2007.

[21] O.L. Mangasarian and E.W. Wild, "Feature Selection in K-median Clustering, "Workshop on Clustering High Dimensional Data and its Applications," La Buena Vista, FL, pp. 23-28, 2004.

[22] J.F. Canny, "A Computational Approach to Edge Detection," IEEE Trans. Pattern Analysis and Machine Int., vol. 8, pp. 679-698, 1986.

[23] JSVM 9.13.1 Software, downloaded from CVS Server, garcon.ient.rwth-aachen.de/cvs/jv

[24] International Telecommunication Union/ITU Radio Communication Sector, "Methodology for the Subjective Assessment of the Quality of Television Pictures," ITU-R BT.500-11, Jan. 2002.

[25] International Telecommunication Union/ITU Radio Communication Sector, "Methodology for the Subjective Assessment of the Quality of Television Pictures,” ITU-R BT.500-10, Mar. 2000.

[26] C.T.E.R. Hewage, S. Worrall, S. Dogan, and A.M. Kondoz, "Quality Evaluation of Colour plus Depth Map Based Stereoscopic Video", IEEE Journal of Selected Topics in Signal Processing: Visual Media Quality Assessment, vol. 3, pp. 304-318, Apr. 2009.

[27] G. J. Burton, S. Nagshineh, and K. H. Ruddock, Processing by the Human Visual System of the Light and Dark Contrast Components of the Retinal Image," Springer Biological Cybernetics, vol. 27, no. 4, pp. 189-197, 1977.

[28] G. Nur, H. Kodikara Arachchi, S. Dogan, and A.M. Kondoz, "Ambient Illumination as a Context for Video Bit Rate Adaptation Decision Taking", IEEE TCSVT, vol. 20, no. 12, pp. 1887-1891, Dec. 2010.

[29] S. Ichihara, N. Kitagawa, and H. Akutsu, "Contrast and Depth perception: Effects of Texture Contrast and Area Contrast," Perception, vol. 36, no. 5, pp. 686-695, 2007.

[30] G. Nur, S. Dogan, H. Kodikara Arachchi, and A. M. Kondoz, "Assessing the Effects of Ambient Illumination Change in Usage Environment on 3D Video Perception for User Centric Media Access and Consumption," 2nd Int. ICST Conf. on User Centric Media, Palma de Mallorca, Spain, 1-3 Sep. 2010.

[31] http://www.xrite.com, Gretag Macbeth Eye-One Display 2

[32] V. Jones, "Mean Direction and Mean Absolute Deviation," ASTM Standards and Eng. Dig. Library, Jan. 2009.

[33] http://www.mathworks.com/access/helpdesk/help/toolbox/curvefit/ 